Rev. Int. Contam. Ambie. 38, 37-56, 2022

https://doi.org/10.20937/RICA.54092

\title{
EVALUACIÓN DE LA CALIDAD DEL AGUA Y DE LA RIBERA EN LA CUENCA DEL RÍO MARGARITAS, CHIAPAS, MÉXICO
}

Assessment the water quality and the riparian quality in the Margaritas river basin, Chiapas, Mexico

\begin{abstract}
Raisa Yarina ESCALONA-DOMENECH ${ }^{1}$, Dulce INFANTE-MATA ${ }^{1 *}$, José Rubén GARCÍA-ALFARO ${ }^{1}$, Neptalí RAMÍREZ-MARCIAL ${ }^{2}$, Claudia Irene ORTIZ-ARRONA ${ }^{3}$ y Everardo BARBA MACÍAS ${ }^{4}$
\end{abstract}

${ }^{1}$ Departamento de Ciencias de la Sustentabilidad, El Colegio de la Frontera Sur, Unidad Tapachula, km 2.5 Carretera Antiguo Aeropuerto, 30700 Tapachula de Córdova y Ordóñez, Chiapas, México.

${ }^{2}$ Departamento de Conservación de la Biodiversidad, Unidad San Cristóbal de Las Casas, Carretera Panamericana y Periférico Sur s/n, Barrio María Auxiliadora, 29290 San Cristóbal de las Casas, Chiapas, México.

${ }^{3}$ Departamento de Ecología y Recursos Naturales, Centro Universitario de la Costa Sur, Universidad de Guadalajara, Avenida Independencia Nacional 151, 48900 Autlán de Navarro, Jalisco, México.

${ }^{4}$ Departamento de Ciencias de la Sustentabilidad, Unidad Villahermosa, km 15.5 Carretera a Reforma, Ranchería Guineo 2da. Sección, 86280 Villahermosa, Tabasco, México.

*Autora para correspondencia: dinfante@ecosur.mx

(Recibido: julio de 2020; aceptado: abril de 2021)

Palabras clave: bioindicador, BMWP, contaminación, RQI, vegetación ribereña

\section{RESUMEN}

El deterioro de la calidad del agua en los ecosistemas acuáticos constituye un problema en todo el mundo, de manera que los recursos hídricos de buena calidad podrían volverse escasos en el futuro. En la cuenca del río Margaritas diversas actividades antrópicas como la agricultura y la ganadería pueden influir en la calidad del agua y las riberas. Esta investigación tuvo como objetivo evaluar la calidad del agua y las riberas en esta cuenca del sureste de México. Se emplearon el Índice de Calidad de las Riberas (RQI, por sus siglas en inglés) y el Î́ndice del Grupo de Trabajo de Monitoreo Biológico (BMWP) adaptado para Costa Rica (BMWP_CR). Se determinaron parámetros físicos y químicos ( $\mathrm{pH}$, oxígeno disuelto, temperatura y conductividad) e hidrológicos (velocidad de la corriente y el caudal) del agua y se recolectaron macroinvertebrados acuáticos en temporadas de secas y lluvias. La calidad ribereña varió de "mala" a "muy buena". Los atributos del RQI más deteriorados fueron la regeneración natural y la composición y estructura de la vegetación ribereña. El índice BMWP_CR mostró que la calidad del agua disminuyó en la temporada de lluvias. Se contabilizó un total de 17676 macroinvertebrados (10 954 en la temporada de secas y 6722 en lluvias) pertenecientes a 13 órdenes y 50 familias. La familia con mayor cantidad de individuos fue Baetidae, seguida de Hydropsychidae y Elmidae. La riqueza y la diversidad biológica ( $\left.\mathrm{H}^{\prime}\right)$ fueron mayores en secas. Este trabajo es el primero en integrar la calidad del agua, la calidad de las riberas y la diversidad de macroinvertebrados a escala local y regional, lo que representa la línea de base en la gestión futura del agua en la zona y para la creación de un índice BMWP adaptado a esta región del sureste de México.

Key words: bioindicator, BMWP, contamination, riparian vegetation, RQI 


\begin{abstract}
Deteriorating water quality is a worldwide problem, so that good quality water resources may become scarce in the future, putting their use for drinking clear water and economic development at risk. In the Margaritas river basin, anthropogenic activities such as agriculture and livestock may be influencing the quality of the water and the banks, so we aimed to assess the quality of the riverbank and the water quality in this basin. The Riparian Quality Index (RQI) and the Index of the Biological Monitoring Working Group (BMWP) adapted for Costa Rica (BMWP_CR) were used; also physical and chemical parameters of the water ( $\mathrm{pH}$, dissolved oxygen, temperature and conductivity) and hydrological parameters (current speed and flow) in dry and rainy seasons were determined. The riparian quality ranged from "bad" to "very good". The attributes of the index with the greatest affectations were the natural regeneration, the composition and structure of the riparian vegetation. The BMWP_CR water quality index showed that water quality decreased in the rainy season. A total of 17676 macroinvertebrate individuals were counted, belonging to 13 orders and 50 families. Baetidae was the family with the largest number of collected individuals, followed by Hydropsychidae and Elmidae. The richness and diversity ( $\left.\mathrm{H}^{\prime}\right)$ values were higher in the dry season. This work is the first to integrate the quality of the water, the quality of the banks and the biodiversity of macroinvertebrates at the local and regional scales, which represents the baseline for future water management in the area and the creation of a BMWP index adapted to this region of southeastern Mexico.
\end{abstract}

\section{INTRODUCCIÓN}

En los ríos, la condición ecológica está relacionada con el estado de calidad del agua, el hábitat, la diversidad y abundancia de organismos acuáticos, los procesos ecológicos y la hidrología (Karr 1999). Diversos indicadores son evaluados para determinar la condición ecológica de los ríos; entre ellos se encuentran los parámetros físicos y químicos que determinan la calidad del agua (Arriaga-Gaona 2009), el uso de organismos acuáticos e índices bióticos (Armitage et al. 1983, Alba-Tercedor y Sánchez-Ortega 1988), la evaluación del hábitat (Pardo et al. 2002), la vegetación ribereña (Magdaleno et al. 2010) y la calidad de las riberas (González del Tánago y García de Jalón 2011).

Las zonas ribereñas, que constituyen uno de los componentes esenciales de las cuencas hidrográficas, se definen como la transición entre el ambiente acuático y el terrestre (Naiman y Décamps 1997). Sus funciones son ser fuente de alimento, regular la temperatura del agua, filtrar nutrientes y contaminantes (Naiman et al. 1993), estabilizar las orillas o márgenes de los ríos (Hood y Naiman 2000) y ser corredores de biodiversidad (Naiman y Décamps 1997). Estas funciones explican por qué el estado de conservación de las riberas es parte importante para la gestión y protección de los recursos hídricos en las cuencas hidrográficas (Cotler 2007). Una forma de caracterizar las riberas en términos de conserva- ción es mediante índices (p. ej., el índice de calidad ecológica de las riberas [RQI; González del Tánago y García de Jalón 2011]). El RQI es fácil de aplicar en campo y se calcula a partir de diferentes atributos riparios para un tramo de río dado (González del Tánago y García de Jalón 2011). La disminución de la calidad de las riberas y el deterioro de su vegetación puede aumentar la cantidad de sólidos disueltos en el agua y afectar la penetración de luz, aumentar la temperatura del agua y contribuir a una eutrofización excesiva (Baillie et al. 2005). Por tanto, se afectan directa e indirectamente la calidad del agua y las comunidades acuáticas asociadas (Fierro et al. 2017).

Existen numerosas causas que influyen en la calidad del agua en las cuencas hidrográficas, como los procesos naturales (p. ej., meteorización, precipitación, erosión del suelo), actividades antrópicas (p. ej., actividades agrícolas, urbanas e industriales) y la mayor utilización de los recursos hídricos (Tyagi et al. 2013). Por estas causas el deterioro de la calidad del agua se ha convertido en un problema grave en todo el mundo y los recursos hídricos de buena calidad podrían escasear en el futuro, lo que pone en riesgo su uso, especialmente para agua potable y desarrollo económico (Vorosmarty et al. 2010).

La disminución de la calidad del agua repercute en la composición y distribución de las comunidades acuáticas (Roldán 1999). Los efectos de la mala calidad del agua sobre los organismos acuáticos 
transcurren con el tiempo, de respuestas individuales (bioquímicas y fisiológicas) a respuestas poblacionales, comunitarias y ecosistémicas (Roldán 1999). Asimismo, la magnitud de los cambios registrados en los organismos depende del tiempo que dure la perturbación de las condiciones iniciales del sistema acuático, de su intensidad y naturaleza (Pinilla 2000). Por ello el empleo de métodos para la evaluación de la calidad del agua en todo el mundo a través de las comunidades biológicas ha aumentado considerablemente (Damanik-Ambarita et al. 2016).

El uso de organismos como indicadores de la calidad del agua es muy popular, especialmente en los países en desarrollo, debido a que no requiere equipos costosos o muy sofisticados, lo que reduce los costos operativos (Ruiz-Picos et al. 2017). También son muy empleados índices bióticos para el monitoreo rutinario de la calidad del agua (Damanik-Ambarita et al. 2016). En este sentido, los macroinvertebrados acuáticos (MA) son bioindicadores útiles (Roldán 1999) debido a su amplia distribución, alta abundancia, muestreo fácil y respuesta rápida a la presión antrópica (Ruiz-Picos et al. 2017).

Los MA muestran un amplio espectro de tolerancia a los contaminantes, que abarcan desde grupos especialmente sensibles hasta aquellos que pueden tolerar condiciones altamente contaminadas (Chang et al. 2014). Las respuestas de los MA incluyen una fuerte reducción de las especies y abundancia en áreas impactadas con predominio de especies tolerantes al disturbio, mientras que las especies sensibles sólo están presentes en los ambientes con menor impacto (Ruiz-Picos et al. 2017). Los MA constituyen uno de los indicadores biológicos más utilizados y tienen una amplia aceptación debido a que simplifican las respuestas complejas que puede brindar una comunidad indicadora, al convertirlas en valores numéricos fácilmente interpretables mediante el uso de índices bióticos (Alonso y Camargo 2005).

Los índices bióticos han sido ampliamente utilizados para la evaluación de la calidad del agua, en especial de los ríos (Springer 2010). Se conocen más de 100 índices, el $60 \%$ de los cuales utilizan macroinvertebrados como bioindicadores (CzerniawskaKusza 2005). En esta gran variedad, el índice del Grupo de Trabajo de Monitoreo Biológico (BMWP, por su sigla en inglés), desarrollado originalmente en el Reino Unido (Armitage et al. 1983), es uno de los más utilizados. Debido a su facilidad de uso y bajo costo, este índice se ha extendido a países de África, Asia, Oceanía y América Latina, donde ha sido adaptado, ya que pueden existir diferencias importantes en la composición taxonómica y las condiciones antrópicas entre los diferentes países (e.g., Chang et al. 2014).

La contaminación es uno de los principales problemas que presentan los cuerpos de agua en México, situación que se ha agravado por el reconocimiento tardío de este deterioro, por lo que los problemas de calidad del agua en el país son graves (Espinal-Carreón et al. 2013). En el estado de Chiapas, en la cuenca media y baja del río Margaritas se observa un cambio en el patrón de uso del suelo, intensificado por actividades productivas como la agricultura y la ganadería. Esta cuenca tiene una extensión total de 19475 ha, de las cuales el $37.5 \%$ (7320.17 ha) corresponden a pastizales inducidos, dedicadas a la actividad ganadera (fundamentalmente ganado bovino y vacuno) y cerca del $1 \%$ se usa para la agricultura de temporada, y el cultivo de mango y la palma africana (INEGI 2013). Estas actividades han provocado la fragmentación y eliminación de la vegetación ribereña (Tovilla 2005), lo cual debe influir en la calidad de las riberas y la calidad del agua, con la subsecuente disminución de la condición ecológica en la cuenca. Sin embargo, hasta el momento no existe ningún estudio científico sobre el tema.

En este contexto, la presente investigación evaluó la calidad de la ribera y la calidad del agua en la cuenca del río Margaritas, a través de dos índices reconocidos por su fácil aplicación en campo y bajo costo (González del Tánago y García de Jalón 2011, Ruiz-Picos et al. 2017): el Índice de Calidad de las Riberas (RQI) y el índice BMWP adaptado para Costa Rica (BMWP_CR). Además, se determinaron parámetros físicos y químicos e hidrológicos del agua en temporadas de secas y lluvias. Los resultados de este estudio serán relevantes para el establecimiento de prioridades de conservación y la restauración de zonas degradadas de la cuenca; además, se podrán emplear para respaldar la gestión futura del agua en la cuenca del río Margaritas.

\section{MATERIALES Y MÉTODOS}

\section{Área de estudio}

La cuenca del río Margaritas se ubica entre los $93^{\circ}$ $07^{\prime} 57^{\prime \prime}$ y $92^{\circ} 59^{\prime} 06^{\prime \prime}$ de longitud oeste y $15^{\circ} 25^{\prime} 01^{\prime \prime}$ y $15^{\circ} 41^{\prime} 40^{\prime \prime}$ de latitud norte y tiene una extensión de 19475.81 ha (Fig. 1). Se encuentra en la región hidrológica 23 Costa de Chiapas (CONAGUA 2009), en el municipio de Pijijiapan. El clima predominante es cálido húmedo (García 1998), la temperatura me- 


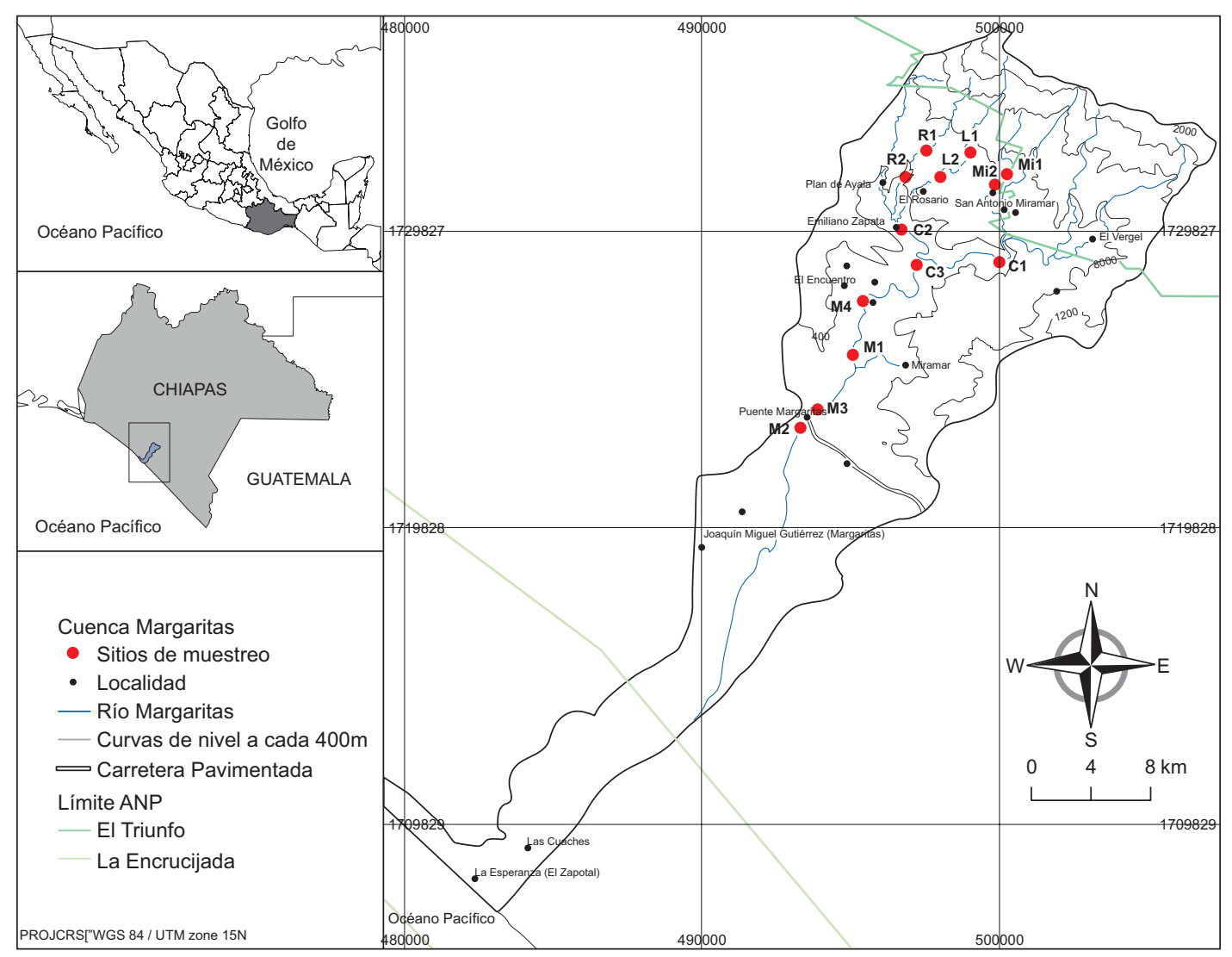

Fig 1. Localización del área de estudio y los sitios muestreados en la cuenca del río Margaritas, Chiapas, México.

dia anual es de $27.6^{\circ} \mathrm{C}$ y el promedio de precipitación anual es de $2596 \mathrm{~mm}$ (datos de la estación meteorológica 23018 de la Comisión Nacional del Agua [CONAGUA]). Los suelos predominantes de la cuenca son los cambisoles, litosoles y regosoles (INEGI 2002). El rango de altitud va desde el nivel del mar hasta los $1800 \mathrm{msnm}$. En las zonas altas (500-650 m) la vegetación corresponde a selva alta perennifolia y selva mediana subperennifolia, mientras que en las zonas media y baja a vegetación secundaria y pastizales tanto cultivados como inducidos, relacionados con actividades agrícolas y pecuarias (INEGI 2013).

Dentro de la cuenca del río Margaritas la población humana total asciende a 5508 habitantes pertenecientes al municipio de Pijijiapan. La localidad con mayor cantidad de población (1581 habitantes de acuerdo con el XIV Censo de Población y Vivienda 2020) es Joaquín Miguel Gutiérrez, conocida popularmente como Margaritas. Otras comunidades cercanas a los sitios de muestreo son: Plan de Ayala, San Antonio Miramar y Emiliano Zapata (Fig. 1). El estudio de la calidad del agua y de la ribera en la cuenca del río Margaritas es relevante si se tiene en cuenta que este río nace en la Sierra Madre de Chiapas, en cuyas zonas alta y baja se encuentran las áreas naturales protegidas Reserva de la Biosfera El Triunfo y Reserva de la Biosfera La Encrucijada, respectivamente, las cuales son zonas conservadas.

Sin embargo, como se ha mencionado anteriormente, en las zonas media y baja de la cuenca se pueden observar el cambio de uso de suelo y el desarrollo de actividades humanas que han conducido su deterioro, por lo cual la conectividad que genera el río Margaritas entre ambas áreas protegidas se encuentra amenazada. Además, esta cuenca tiene potencialidad para contener una reserva de agua (CONAGUA 2011), por lo que es necesaria la determinación de su caudal ecológico frente a la intensificación de actividades productivas que demandan grandes volúmenes de agua, en perjuicio de la conservación y el uso sustentable de sus recursos naturales. Esta investigación servirá como línea base para ello.

Para el muestreo se seleccionaron 13 sitios en la cuenca del río Margaritas, cuya longitud total es de $21.3 \mathrm{~km}$, de los cuales se muestrearon $11.2 \mathrm{~km}$. Esta 
longitud se obtuvo con la ayuda del software Qgis 3.10 (QGIS 2019). Los sitios de muestreo se seleccionaron según su ubicación en el río (caudal principal, corrientes tributarias, confluencias), la altitud, la cobertura vegetal, los usos del suelo y el orden del río (Cuadro I). Las diferencias geomorfológicas de la zona más baja del río respecto a la zona alta, así como el deterioro y el azolvamiento del lecho del río en las zonas bajas determinaron que no se muestreara en su totalidad.

\section{Calidad de las riberas}

La evaluación de la calidad ecológica de las riberas se realizó a través del índice de calidad de las riberas (RQI) propuesto por González del Tánago y García de Jalón (2011). Este método integra siete atributos de fácil reconocimiento visual, cada uno con valor máximo de 15 puntos: 1) dimensiones del espacio ripario ocupado por vegetación (ancho promedio del corredor ripario), 2) continuidad longitudinal de la vegetación leñosa, 3) composición y estructura de la vegetación ribereña, 4) regeneración natural de las principales especies leñosas, 5) condición de las orillas, 6) conectividad transversal del cauce con sus riberas y llanura de inundación, 7) conectividad vertical a través de la permeabilidad y el grado de alteración de los materiales y relieve de los suelos riparios. Los tres primeros atributos determinan la estructura física del corredor ribereño, mientras que los cuatro restantes están relacionados con el funcionamiento del sistema. La evaluación de los atributos de RQI se realizó de forma sistemática: los atributos 1, 2 y 3 se caracterizaron en cada orilla del río por separado, mientras que los atributos 4 al 7 se caracterizaron conjuntamente en ambos márgenes.

La calidad de los 13 sitios (tramos de $250 \mathrm{~m}$ ) en el río Margaritas se evaluó con el sistema de puntuación de cero a 150, según lo indicado por el protocolo del RQI. La calidad de las riberas en cada sitio se determinó a partir de la puntuación obtenida de la sumatoria de los siete atributos evaluados, con las siguientes categorías: muy bueno (150-130), bueno (129-100), moderado (99-70), pobre (69-40), malo (39-10) y muy malo $(<10)$ (Cuadro II) (González del Tánago y García de Jalón 2011).

\section{Análisis de la calidad del agua Parámetros físicos, químicos e hidrológicos}

El muestreo se realizó en temporada de lluvias (mayo-julio de 2017) y en secas (febrero-abril de 2018). En cada sitio se obtuvieron, directamente en campo y por triplicado, los siguientes parámetros: valor del oxígeno disuelto (OD), conductividad eléctrica (CE), temperatura (T) y $\mathrm{pH}$. Los valores de

CUADRO I. CARACTERÍSTICAS FÍSICAS DE LOS SITIOS DE ESTUDIO EN LA CUENCA DEL RÍO MARGARITAS, CHIAPAS, MÉXICO, ORDENADOS DE MAYOR A MENOR ALTITUD.

\begin{tabular}{|c|c|c|c|c|}
\hline $\begin{array}{l}\text { Nombre del } \\
\text { intervalo }\end{array}$ & Río & $\begin{array}{l}\text { Altitud } \\
(\mathrm{msnm})\end{array}$ & $\begin{array}{l}\text { Orden } \\
\text { del río }\end{array}$ & $\begin{array}{l}\text { Cobertura vegetal/uso del suelo adyacente a } \\
\text { la ribera }\end{array}$ \\
\hline Loco uno (L1) & $\mathrm{T}$ & 652 & 4 & Selva/selva. \\
\hline La Mina1 (Mi1) & $\mathrm{T}$ & 612 & 4 & Selvas/selva. \\
\hline Loco dos (L2) & $\mathrm{T}$ & 476 & 4 & Pastizal-vegetación secundaria/ganadería. \\
\hline La Mina dos (Mi2) & $\mathrm{T}$ & 472 & 4 & Pastizal-vegetación secundaria/vivero forestal. \\
\hline Ramón uno (R1) & $\mathrm{T}$ & 460 & 4 & Selva/selva. \\
\hline Ramón dos (R2) & $\mathrm{T}$ & 386 & 4 & Pastizal/poblado, ganadería. \\
\hline Primera Confluencia $(\mathrm{C} 1)$ & $\mathrm{P}$ & 347 & 6 & $\begin{array}{l}\text { Selva-vegetación secundaria/ganadería, } \\
\text { camino. }\end{array}$ \\
\hline Segunda Confluencia (C2) & $\mathrm{T}$ & 319 & 5 & Pastizal/poblado, camino. \\
\hline Tercera Confluencia (C3) & $\mathrm{P}$ & 250 & 6 & Pastizal-vegetación secundaria/ganadería. \\
\hline Margarita cuatro (M4) & $\mathrm{P}$ & 168 & 6 & $\begin{array}{l}\text { Pastizal-vegetación secundaria/área recreativa, } \\
\text { camino. }\end{array}$ \\
\hline Margarita uno (M1) & $\mathrm{P}$ & 128 & 6 & Pastizal-vegetación secundaria/camino. \\
\hline Margarita tres (M3) & $\mathrm{P}$ & 68 & 6 & $\begin{array}{l}\text { Pastizal/poblado, ganadería, área recreativa, } \\
\text { camino, puente de autopista, bordos para } \\
\text { evitar la inundación. }\end{array}$ \\
\hline Margarita dos (M2) & $\mathrm{P}$ & 56 & 6 & $\begin{array}{l}\text { Pastizal/poblado, ganadería, puente de auto- } \\
\text { pista, bordos para evitar la inundación. }\end{array}$ \\
\hline
\end{tabular}

P: río principal, T: río tributario. 
CUADRO II. INTERPRETACIÓN DE LOS VALORES TOTALES DEL RQI*.

\begin{tabular}{|c|c|c|}
\hline RQI & Estado de la ribera & Opciones de manejo \\
\hline $150-130$ & Muy bueno & $\begin{array}{l}\text { Los atributos riparios están en sus condiciones naturales, sin ningún tipo de amenazas } \\
\text { en su funcionamiento. } \\
\text { Gran interés en su conservación y protección, para mantener el estado actual y prevenir } \\
\text { alteraciones futuras en el sistema ripario. }\end{array}$ \\
\hline $129-100$ & Bueno & $\begin{array}{l}\text { La mayoría de sus atributos se encuentran en muy buenas condiciones y uno o dos } \\
\text { pueden estar alterados. Es necesario aplicar medidas de protección para prevenir nuevos } \\
\text { impactos y medidas de restauración para lograr la integridad optima en las funciones } \\
\text { riparias. Eliminar presiones e impactos en la mayor medida posible. }\end{array}$ \\
\hline $99-70$ & Moderado (Regular) & $\begin{array}{l}\text { Muchos de sus atributos están moderadamente afectados. } \\
\text { El sistema ripario necesita medidas de restauración que aseguren el correcto funcio- } \\
\text { namiento hidrológico y ecológico. Eliminando o reduciendo presiones e impactos en } \\
\text { la mayor medida posible. }\end{array}$ \\
\hline $69-40$ & Pobre & $\begin{array}{l}\text { Gran número de los atributos están moderadamente afectados. } \\
\text { El sistema ripario necesita medidas de rehabilitación o restauración, para así mejorar u } \\
\text { recuperar las funciones hidrológicas y ecológicas. Reducir presiones e impactos lo más } \\
\text { posible y diseñar medidas de compensación para mejorar las condiciones ambientales. }\end{array}$ \\
\hline $39-10$ & Malo & $\begin{array}{l}\text { Muchos atributos en mal estado (alterados). } \\
\text { El sistema ripario necesita nuevas estrategias de trabajo de rehabilitación o restaura- } \\
\text { ción, para así reintroducir o mejorar gradualmente las funciones riparias hidrológicas } \\
\text { y ecológicas. Reduciendo las presiones e impactos lo más que se pueda y mejorar la } \\
\text { precepción social acerca de la degradación del río. }\end{array}$ \\
\hline$<10$ & Muy malo & $\begin{array}{l}\text { Casi todos sus atributos están sumamente alterados. } \\
\text { El sistema ripario necesita nuevos trabajos de rehabilitación o remediación, para así } \\
\text { rescatar y reintroducir las funciones riparias. Mejorar las condiciones ambientales } \\
\text { para lograr un estado potencialmente bueno y aumentar la percepción social sobre la } \\
\text { degradación de los ríos. }\end{array}$ \\
\hline
\end{tabular}

*González del Tánago y García de Jalón (2011).

RQI: Índice de Calidad de la Ribera (Riparian Quality Index).

los tres primeros se obtuvieron con una sonda multiparamétrica YSI modelo 85, mientras que el valor del pH se determinó con un sensor Eco Test modelo $\mathrm{pH} 2$. Estos parámetros se seleccionaron porque ejercen una influencia importante sobre la distribución de los macroinvertebrados (Vivas et al. 2002) y a menudo son los factores a los que son más sensibles los organismos (Roldán 1999); además, porque el OD y el $\mathrm{pH}$ se encuentran entre los indicadores físicos y químicos más utilizados en la valoración de la calidad del agua (Ruiz et al. 2007).

Se calcularon también parámetros hidrológicos como la velocidad de la corriente (VC) y el caudal. La VC se obtuvo a través del método del objeto flotante, midiendo el tiempo que demoró el objeto en recorrer $5 \mathrm{~m}$ de distancia (Wetzel y Likens 2000). El valor del caudal en cada uno de los sitios muestreados se obtuvo a través de la relación existente entre el área de sección transversal del canal y la VC (Wetzel y Likens 2000), empleando la siguientes fórmula:

$$
Q=(w) \times 97(d) \times(a) \times(L / t)
$$

donde $w$ es el ancho (m) de la sección transversal del canal, d es la profundidad media (m), $L / t$ es la VC $(\mathrm{m} / \mathrm{s})$ y $a$ es un factor de corrección y es igual a 0.8 .

\section{Diversidad de macroinvertebrados}

Los macroinvertebrados se recolectaron durante las temporadas de lluvias y secas. Para la recolección se empleó una red Surber de $0.09 \mathrm{~m}^{2}$ de superficie de muestreo y $500 \mu \mathrm{m}$ de apertura de malla, y una red tipo D de $500 \mu \mathrm{m}$ de apertura de malla y superficie de barrido de $10 \mathrm{~m}$. Se hicieron tres repeticiones por cada técnica en cada sitio. Las muestras fueron trasladadas al laboratorio, donde se limpiaron de todo el material acompañante y se conservaron en alcohol al 96 \% (Merritt et al. 2008). Los macroinvertebrados fueron contabilizados e identificados hasta el nivel taxonómico de familias con ayuda de estéreos ZEISS Stemi 2000-C y mediante las claves 
de Wiggins (2000), Thompson (2004) y Merritt et al. (2008). Los especímenes fueron depositados en la colección de referencia de organismos acuáticos de El Colegio de la Frontera Sur, Unidad Villahermosa, Tabasco, México.

La caracterización estructural de los ensambles de macroinvertebrados se realizó por temporadas y por sitios a través del cálculo de la diversidad de especies mediante el índice de Shannon-Wiener (Moreno 2001). Los macroinvertebrados obtenidos con ambos tipos de arte se unificaron para conformar una sola muestra. Para evaluar la relación entre los macroinvertebrados, los sitios de muestreo y los parámetros físicos y químicos del agua, se realizó un análisis de correspondencia canónica (ACC) empleando el programa Past v. 3.21 (Hammer et al. 2012).

\section{Índice de calidad del agua (BMWP_CR)}

Para evaluar la calidad del agua en los sitios muestreados se estimó el índice BMWP_CR (Gutiérrez-Fonseca y Lorion 2014). Este índice es una adaptación del BMWP creado por Armitage et al. (1983) en el Reino Unido. El BMWP es un índice relacionado con la riqueza de taxones familiares, y se basa en dos hipótesis: 1) los cambios en abundancia y biodiversidad de las comunidades de macroinvertebrados se deben a la reducción de los niveles de OD debido a la contaminación orgánica y 2) algunos invertebrados son más sensibles a la contaminación que otros. Con base en esto, las familias sensibles a la contaminación reciben puntajes más altos que las menos tolerantes (Armitage et al. 1983). El BMWP_CR es el índice oficial para monitorear la calidad del agua en Costa Rica (Poder Ejecutivo 2007) y fue seleccionado por las similitudes taxonómicas a nivel de familias de macroinvertebrados entre esta región y el río Margaritas.

El BMWP CR se calculó acumulando los valores de sensibilidad preasignados a la contaminación del agua (Cuadro III) para las distintas familias identificadas en cada sitio. Con base en el resultado de la sumatoria de los valores de cada familia, el índice establece seis niveles de calidad del agua:

1. Excelente $(>120)$.

2. Buena: agua no contaminada ni sensiblemente alterada (101-120).

3. Regular: contaminación moderada (61-100).

4. Mala: agua contaminada (36-60).

5. Mala: agua muy contaminada (16-35).

6. Muy mala: agua extremadamente contaminada $(<15)$.

\section{RESULTADOS}

\section{Calidad de las riberas}

Según el RQI, las condiciones de los sitios variaron de "malas" a "muy buenas". Se encontraron cinco de los seis rangos de calidad del índice (Cuadro IV). Los sitios con mayores valores de RQI fueron L1 y Mi1, los únicos que obtuvieron la condición de "muy bueno". Los sitios con menor calidad de las riberas fueron M3 y M2 clasificados como "malo" y "pobre", respectivamente. Los atributos del índice que presentaron mayores afectaciones fueron la regeneración natural y la composición y estructura de la vegetación ribereña (Cuadro IV). Los sitios con rangos "muy bueno" y "bueno" (L1, Mil, R1, $\mathrm{C} 1$ y Mi2), coinciden en la presencia de vegetación más conservada (selva o combinación de selva con vegetación secundaria) y están en zonas de difícil acceso donde aún no llega la actividad ganadera, muy difundida en la cuenca.

En estos sitios no hay restricciones para el desarrollo y extensión de la vegetación riparia a través del valle, debido a la poca influencia humana y ausencia de especies invasoras. Tampoco hay elementos constructivos o de otra índole que impidan la conectividad longitudinal y vertical del cauce principal con los restantes elementos del sistema fluvial (i.e., riberas, llanura de inundación y el medio hiporreico). Presentan orillas estables sin indicio de erosión y sin alteraciones del relieve y los suelos riparios, lo que permite que sean permeables.

En contraste, los sitios con condición "pobre" y "mala" (R2, C2, M2 y M3, Cuadro IV) coinciden en tener pastizales adyacentes a las riberas (Cuadro I) donde se desarrolla una intensa actividad ganadera y hay algún asentamiento humano (M3, C2, Cuadro I). En estos tramos se produce una disminución de las dimensiones del espacio ripario ocupado por vegetación ribereña, la cual ha sido fragmentada y sustituida con pastos para la alimentación del ganado. Lo anterior implica disminución de la heterogeneidad física del paisaje, por lo que se observan indicios de erosión de las orillas, así como pérdida de la conectividad transversal del cauce con la llanura de inundación y de la conectividad vertical con el medio hiporreico. Los procesos de urbanización y la construcción de caminos y bordos (estos últimos para la protección contra inundaciones), sumados al pisoteo del ganado, han conducido a la compactación del suelo y la modificación del relieve y los materiales naturales del terreno, promoviendo la subsidencia. 
CUADRO III. PUNTUACIÓN ASIGNADA A LAS FAMILIAS DE MACROINVERTEBRADOS IDENTIFICADOS Y CANTIDAD DE INDIVIDUOS EN LOS 13 SITIOS DE MUESTREO EN EL RÍO MARGARITAS.

\begin{tabular}{|c|c|c|c|c|c|}
\hline Clase & Orden & Familia & Abreviatura & $\begin{array}{l}\text { Puntaje según } \\
\text { EL BMWP_CR }\end{array}$ & $\begin{array}{l}\text { Individuos recolec- } \\
\text { tados }\end{array}$ \\
\hline \multirow{47}{*}{ Insecta } & \multirow{6}{*}{ Coleoptera } & Dryopidae & Dry. & 5 & 330 \\
\hline & & Dytiscidae & Dyt. & 4 & 37 \\
\hline & & Elmidae & Elm. & 5 & 2596 \\
\hline & & Gyrinidae & Gyr. & 4 & 1 \\
\hline & & Hydrophilidae & Hyd. & 3 & 4 \\
\hline & & Psephenidae & Pse. & n.a. & 417 \\
\hline & \multirow{10}{*}{ Diptera } & Chironomidae & Chi. & 2 & 36 \\
\hline & & Empididae & Emp. & 4 & 4 \\
\hline & & Ephydridae & Eph. & 2 & 1 \\
\hline & & Muscidae & Mus. & 4 & 3 \\
\hline & & Pelecorhynchidae & Pel. & n.a. & 3 \\
\hline & & Psychodidae & Psy. & 3 & 3 \\
\hline & & Simuliidae & Sim. & 4 & 90 \\
\hline & & Stratiomyidae & Str. & 4 & 20 \\
\hline & & Tabanidae & Tab. & 4 & 93 \\
\hline & & Tipulidae & Tip. & 4 & 47 \\
\hline & \multirow{6}{*}{ Ephemeroptera } & Baetidae & Bae. & 5 & 3686 \\
\hline & & Caenidae & Cae. & 4 & 142 \\
\hline & & Euthyplociidae & Eut. & 6 & 57 \\
\hline & & Heptageniidae & Hep. & 9 & 351 \\
\hline & & Leptohyphidae & Lept. & 5 & 1164 \\
\hline & & Leptophlebiidae & Lep. & 8 & 1339 \\
\hline & \multirow{10}{*}{ Hemiptera } & Belostomatidae & Bel. & 4 & 146 \\
\hline & & Gelastocoridae & Gel. & n.a. & 4 \\
\hline & & Gerridae & Ger. & 6 & 27 \\
\hline & & Hebridae & Heb. & 6 & 7 \\
\hline & & Hydrometridae & Hyd. & n.a. & 6 \\
\hline & & Macrovellidae & Mac. & n.a. & 13 \\
\hline & & Mesoveliidae & Mes. & n.a. & 2 \\
\hline & & Naucoridae & Nau. & 4 & 704 \\
\hline & & Nepidae & Nep. & 4 & 2 \\
\hline & & Veliidae & Vel. & n.a. & 162 \\
\hline & Lepidoptera & Crambidae & Cra. & n.a. & 121 \\
\hline & Megaloptera & Corydalidae & Cor. & 6 & 496 \\
\hline & \multirow{5}{*}{ Odonata } & Calopterygidae & Cal. & 4 & 98 \\
\hline & & Coenagrionidae & Coe. & 4 & 178 \\
\hline & & Gomphidae & Gom. & 7 & 228 \\
\hline & & Libellulidae & Lib. & 6 & 122 \\
\hline & & Platystictidae & Pla. & 7 & 144 \\
\hline & Plecoptera & Perlidae & Per. & 9 & 907 \\
\hline & \multirow{7}{*}{ Trichoptera } & Calamoceratidae & Cal. & 8 & 7 \\
\hline & & Hydrobiosidae & Hyd. & 9 & 15 \\
\hline & & Hydropsychidae & Hydr. & 5 & 2727 \\
\hline & & Leptoceridae & Leptoc. & 8 & 9 \\
\hline & & Philopotamidae & Phi. & 7 & 688 \\
\hline & & Polycentropodidae & Pol. & 6 & 8 \\
\hline & & Psychomyiidae & Psyc. & n.a. & 4 \\
\hline
\end{tabular}

BMWP_CR: Índice del Grupo de Trabajo de Monitoreo Biológico adaptado para Costa Rica. n.a.: valor no asignado a esta familia por el BMWP_CR. 
CUADRO III. PUNTUACIÓN ASIGNADA A LAS FAMILIAS DE MACROINVERTEBRADOS IDENTIFICADOS Y CANTIDAD DE INDIVIDUOS EN LOS 13 SITIOS DE MUESTREO EN EL RÍO MARGARITAS.

\begin{tabular}{lllccr}
\hline Clase & Orden & Familia & Abreviatura & $\begin{array}{c}\text { Puntaje según } \\
\text { EL BMWP_CR }\end{array}$ & $\begin{array}{c}\text { Individuos recolec- } \\
\text { tados }\end{array}$ \\
\hline Gastropoda & Sorbeoconcha & Thiaridae & Thi. & n.a. & 216 \\
\hline \multirow{2}{*}{ Malacostraca } & Decapoda & Palaemonidae & Pal. & n.a. & 190 \\
& & Pseudothelphusidae & Pseudo. & n.a. & 18 \\
\hline & Total & & & 17673 \\
\hline
\end{tabular}

BMWP_CR: Índice del Grupo de Trabajo de Monitoreo Biológico adaptado para Costa Rica. n.a.: valor no asignado a esta familia por el BMWP_CR.

\section{Análisis de la calidad del agua Parámetros físicos y químicos}

Los valores de $\mathrm{pH}$ obtenidos en ambas temporadas de muestreo indican aguas ligeramente básicas en todos los sitios de estudio. Los valores promedios fluctuaron de 7.7 a 8.1 en la temporada de lluvias, y de 7.7 a 8.8 en secas. El valor de la desviación estándar señala que hay poca variabilidad en los datos obtenidos de $\mathrm{pH}$ (Cuadro V). Los valores promedio del OD para la temporada de lluvias fueron ligeramente mayores respecto a los registrados para secas (7.3-8.4 $\mathrm{mg} / \mathrm{L}$ y 5.9-8.4 mg/L, respectivamente) (Cuadro V).

Para todos los sitios, los valores de conductividad eléctrica (CE) registrados fueron mayores en secas que en lluvias, observándose que la CE generalmente fue mayor en los sitios ubicados a menos altitud. El valor promedio mínimo se obtuvo en el sitio L1 (45.2 $\mu \mathrm{S} / \mathrm{cm}$ ) en la temporada de lluvias, mientras que el mayor promedio se registró durante la temporada de secas en el sitio M3 (128.1 $\mu \mathrm{S} / \mathrm{cm})$ (Cuadro V). Los valores encontrados de $\mathrm{T}$ del agua fueron mayores para la temporada de secas que en lluvias, los valores promedios oscilaron entre los $21.2^{\circ} \mathrm{C}$ en el sitio Mil y los $27.3^{\circ} \mathrm{C}$ en el sitio $\mathrm{C} 2$ en la temporada de lluvias, mientras que en secas se registraron valores desde $22.2{ }^{\circ} \mathrm{C}$ en Mi1 y los $29.9^{\circ} \mathrm{C}$ en el sitio M4.

\section{Parámetros hidrológicos}

La velocidad de la corriente (VC) fue mayor en los sitios ubicados a menor altitud (sitios M3, M2, M4) durante la temporada de lluvias, mientras que los valores menores ocurrieron hacia la parte alta (sitios Mi2, L1, L2) durante la temporada de secas (Cuadro V). Los valores obtenidos en ambas temporadas son muy contrastantes (Cuadro V).

De forma general, el caudal aumentó aguas abajo. El rango de valores fluctuó entre $10.2 \mathrm{~m}^{3} / \mathrm{s}$ en M3 en la temporada de lluvias y $0.1 \mathrm{~m}^{3} / \mathrm{s}$ en los sitios Mi2, L1 y R2 en la temporada de secas (Cuadro V). Estos resultados indican la influencia de la temporada de muestreo (lluvias-secas) en los valores del caudal. Los mayores valores se obtuvieron en lluvias para todos los sitios.

\section{Diversidad de macroinvertebrados e índice de cali- dad del agua (BMWP_CR)}

Se contabilizaron un total de 17676 organismos (10 954 en la temporada de secas y 6722 en lluvias), pertenecientes a 13 órdenes y 50 familias (Cuadro III). La clase Insecta fue la más representada con 11 órdenes y 47 familias (Cuadro III). La familia con mayor cantidad de individuos recolectados fue Baetidae con 3686 individuos, seguida por Hydropsychidae con 2727 y Elmidae con 2596 (Fig. 2). El sitio con mayor cantidad de individuos recolectados fue C3 (3319, 2398 en secas y 921 en lluvias), seguido de C1 (1719 individuos, 963 en secas y 756 en lluvias) y M1 con 1606 individuos (1397 en secas y 209 en lluvias) (Cuadro III).

La riqueza y diversidad biológica $\left(\mathrm{H}^{\prime}\right)$ representada por el número de familias de macroinvertebrados, sugieren que en la temporada de secas hay mejores índices de diversidad, equidad y abundancia, y los valores entre sitios fueron menos contrastantes que en la temporada de lluvias (Cuadro VI).

En cuanto al índice de calidad de agua, se observa variación entre las dos temporadas de muestreo (lluvias-secas), ya que los valores de calidad del agua (BMWP_CR) en secas son mayores que en lluvias (Cuadro VI). En la temporada de secas, los sitios Mi1, C1 y L2 (en ese orden) presentaron mejor calidad del agua mientras que M3 y M2 se clasificaron con calidad del agua regular. Durante la temporada de lluvias los sitios con mejor calidad del agua fueron 


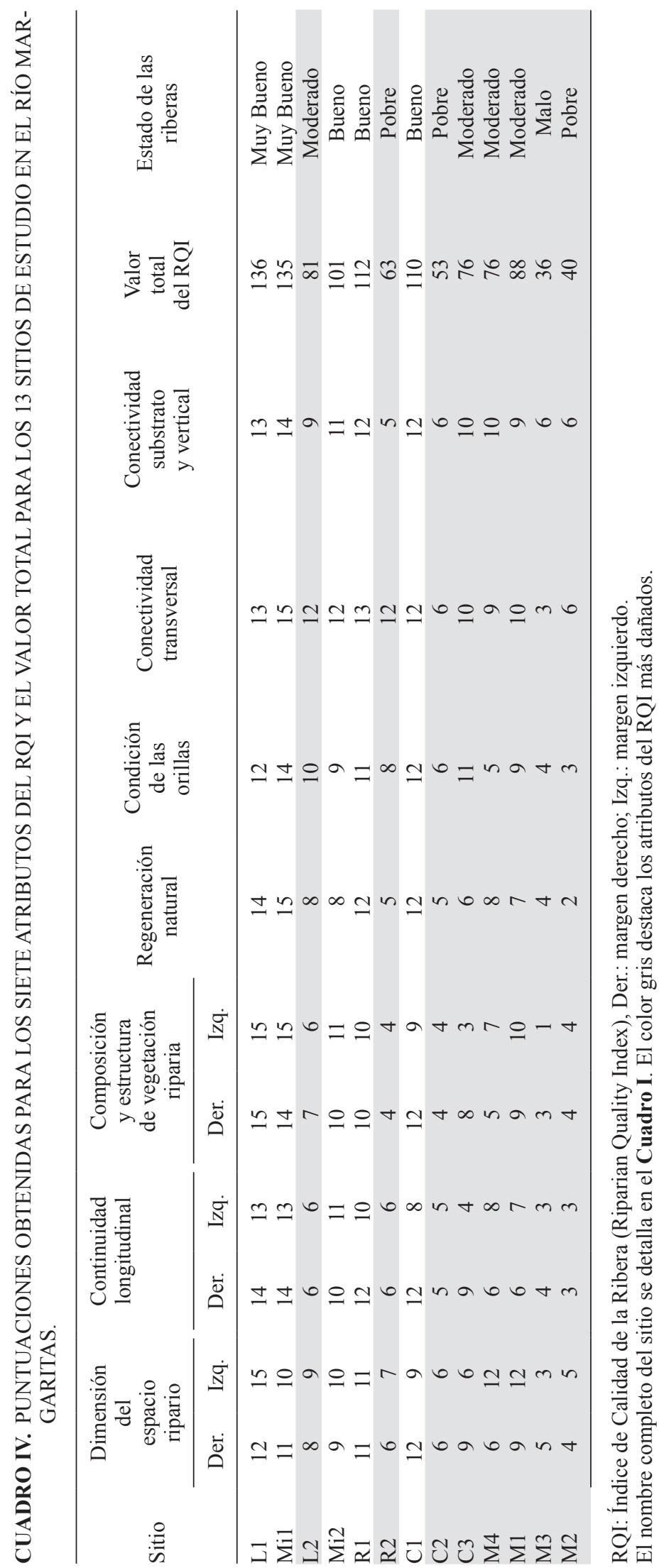


CUADRO V. VARIACIÓN DE LOS PARÁMETROS FÍSICOS Y QUÍMICOS DEL AGUA Y DE LOS PARÁMETROS HIDROLÓGICOS EN LOS SITIOS MUESTREADOS DEL RÍO MARGARITAS. SE MUESTRA LA MEDIA Y LA DESVIACIÓN ESTÁNDAR DE CADA PARÁMETRO EN LAS TEMPORADAS DE LLUVIAS Y DE SECAS.

\begin{tabular}{|c|c|c|c|c|c|c|c|}
\hline \multirow{3}{*}{ Sitios } & \multirow{3}{*}{ Temporada } & \multicolumn{6}{|c|}{ Parámetros } \\
\hline & & \multicolumn{4}{|c|}{ Físicos y químicos } & \multicolumn{2}{|c|}{ Hidrológicos } \\
\hline & & $\mathrm{pH}$ & $\begin{array}{c}\mathrm{OD} \\
(\mathrm{mg} / \mathrm{L})\end{array}$ & $\begin{array}{c}\mathrm{CE} \\
(\mu \mathrm{S} / \mathrm{cm})\end{array}$ & $\begin{array}{c}\mathrm{T} \\
\left({ }^{\circ} \mathrm{C}\right)\end{array}$ & $\begin{array}{c}\mathrm{VC} \\
(\mathrm{m} / \mathrm{s})\end{array}$ & $\begin{array}{l}\text { Caudal } \\
\left(\mathrm{m}^{3} / \mathrm{s}\right)\end{array}$ \\
\hline \multirow{2}{*}{ L1 } & $\mathrm{Ll}$ & $7.9 \pm 0.20$ & $7.7 \pm 0.58$ & $45.2 \pm 3.63$ & $22.1 \pm 0.90$ & $0.5 \pm 0.10$ & $1.5 \pm 0.01$ \\
\hline & $\mathrm{S}$ & $8.0 \pm 0.38$ & $6.3 \pm 0.80$ & $75.2 \pm 7.14$ & $22.9 \pm 0.74$ & $0.04 \pm 0.01$ & $0.1 \pm 0.01$ \\
\hline \multirow{2}{*}{ Mil } & $\mathrm{Ll}$ & $7.9 \pm 0.17$ & $8.3 \pm 0.12$ & $50.5 \pm 1.76$ & $21.2 \pm 0.00$ & $0.7 \pm 0.10$ & $1.4 \pm 0.01$ \\
\hline & $\mathrm{S}$ & $7.8 \pm 0.45$ & $7.8 \pm 0.50$ & $73.0 \pm 10.89$ & $22.2 \pm 1.30$ & $0.3 \pm 0.01$ & $0.3 \pm 0.00$ \\
\hline \multirow{2}{*}{ L2 } & Ll & $7.7 \pm 0.24$ & $7.3 \pm 0.47$ & $70.9 \pm 11.21$ & $24.6 \pm 1.83$ & $0.3 \pm 0.10$ & $0.4 \pm 0.01$ \\
\hline & $\mathrm{S}$ & $7.9 \pm 0.43$ & $6.4 \pm 1.08$ & $89.6 \pm 9.01$ & $27.2 \pm 1.36$ & $0.2 \pm 0.00$ & $0.2 \pm 0.01$ \\
\hline \multirow{2}{*}{ Mi2 } & $\mathrm{Ll}$ & $7.9 \pm 0.17$ & $8.0 \pm 0.11$ & $65.4 \pm 1.14$ & $23.5 \pm 0.12$ & $0.7 \pm 0.08$ & $0.73 \pm 0.01$ \\
\hline & $\mathrm{S}$ & $7.7 \pm 0.40$ & $7.1 \pm 0.42$ & $87.1 \pm 4.35$ & $24.5 \pm 0.90$ & $0.2 \pm 0.03$ & $0.1 \pm 0.01$ \\
\hline \multirow{2}{*}{ R1 } & $\mathrm{Ll}$ & $7.8 \pm 0.17$ & $7.6 \pm 0.30$ & $57.7 \pm 6.62$ & $23.5 \pm 0.88$ & $0.8 \pm 0.06$ & $1.0 \pm 0.02$ \\
\hline & $\mathrm{S}$ & $8.0 \pm 0.37$ & $6.7 \pm 1.28$ & $81.1 \pm 15.25$ & $23.7 \pm 0.65$ & $0.4 \pm 0.01$ & $0.2 \pm 0.01$ \\
\hline \multirow{2}{*}{ R2 } & $\mathrm{Ll}$ & $7.9 \pm 0.18$ & $7.9 \pm 0.46$ & $57.5 \pm 4.31$ & $24.7 \pm 0.54$ & $0.3 \pm 0.1$ & $0.5 \pm 0.01$ \\
\hline & $\mathrm{S}$ & $7.7 \pm 0.38$ & $6.6 \pm 0.71$ & $106.8 \pm 15.37$ & $26.1 \pm 0.53$ & $0.2 \pm 0.06$ & $0.1 \pm 0.01$ \\
\hline \multirow{2}{*}{$\mathrm{C} 1$} & $\mathrm{Ll}$ & $7.9 \pm 0.15$ & $8.3 \pm 0.21$ & $69.0 \pm 12.98$ & $22.3 \pm 0.66$ & $0.6 \pm 0.15$ & $1.8 \pm 0.01$ \\
\hline & $\mathrm{S}$ & $8.3 \pm 0.00$ & $7.6 \pm 0.68$ & $114.0 \pm 0.74$ & $26.9 \pm 0.06$ & $0.4 \pm 0.04$ & $0.5 \pm 0.01$ \\
\hline \multirow{2}{*}{$\mathrm{C} 2$} & $\mathrm{Ll}$ & $7.7 \pm 0.15$ & $7.7 \pm 0.80$ & $81.5 \pm 0.35$ & $23.9 \pm 0.10$ & $0.5 \pm 0.05$ & $0.9 \pm 0.02$ \\
\hline & $\mathrm{S}$ & $7.9 \pm 0.25$ & $7.0 \pm 0.43$ & $97.6 \pm 1.33$ & $27.3 \pm 0.00$ & $0.4 \pm 0.03$ & $0.6 \pm 0.03$ \\
\hline \multirow{2}{*}{$\mathrm{C} 3$} & Ll & $7.8 \pm 0.12$ & $8.4 \pm 0.21$ & $80.2 \pm 0.67$ & $24.0 \pm 0.10$ & $1.0 \pm 0.03$ & $4.8 \pm 0.10$ \\
\hline & $\mathrm{S}$ & $8.2 \pm 0.10$ & $8.4 \pm 0.46$ & $102.3 \pm 3.98$ & $25.6 \pm 0.12$ & $0.6 \pm 0.08$ & $2.3 \pm 0.02$ \\
\hline \multirow{2}{*}{ M4 } & $\mathrm{Ll}$ & $8.1 \pm 0.18$ & $7.3 \pm 0.42$ & $79.9 \pm 3.95$ & $26.2 \pm 0.79$ & $1.06 \pm 0.05$ & $6.8 \pm 0.1$ \\
\hline & $\mathrm{S}$ & $8.4 \pm 0.06$ & $6.8 \pm 0.33$ & $115.9 \pm 2.63$ & $29.9 \pm 0.10$ & $0.7 \pm 0.02$ & $1.3 \pm 0.01$ \\
\hline \multirow{2}{*}{ M1 } & $\mathrm{Ll}$ & $8.0 \pm 0.26$ & $8.1 \pm 0.37$ & $76.7 \pm 2.38$ & $24.6 \pm 0.51$ & $0.8 \pm 0.1$ & $4.6 \pm 0.1$ \\
\hline & $\mathrm{S}$ & $8.8 \pm 0.06$ & $5.9 \pm 0.47$ & $119.0 \pm 1.23$ & $27.9 \pm 0.21$ & $0.7 \pm 0.03$ & $3.7 \pm 0.1$ \\
\hline \multirow{2}{*}{ M3 } & $\mathrm{Ll}$ & $8.0 \pm 0.18$ & $7.8 \pm 0.19$ & $81.5 \pm 5.66$ & $25.8 \pm 0.73$ & $1.5 \pm 0.1$ & $10.2 \pm 0.05$ \\
\hline & $\mathrm{S}$ & $8.8 \pm 0.00$ & $6.9 \pm 0.21$ & $128.1 \pm 0.47$ & $28.8 \pm 0.12$ & $0.9 \pm 0.05$ & $3.0 \pm 0.06$ \\
\hline \multirow{2}{*}{ M2 } & $\mathrm{Ll}$ & $8.0 \pm 0.18$ & $7.9 \pm 0.17$ & $79.2 \pm 3.91$ & $25.1 \pm 1.02$ & $1.1 \pm 0.1$ & $7.5 \pm 0.08$ \\
\hline & $\mathrm{S}$ & $8.4 \pm 0.17$ & $7.4 \pm 0.27$ & $117.3 \pm 2.66$ & $26.3 \pm 0.06$ & $0.5 \pm 0.1$ & $2.1 \pm 0.01$ \\
\hline
\end{tabular}

L1: lluvias; S: secas; OD: oxígeno disuelto; CE: conductividad eléctrica; T: temperatura; VC: velocidad de la corriente.

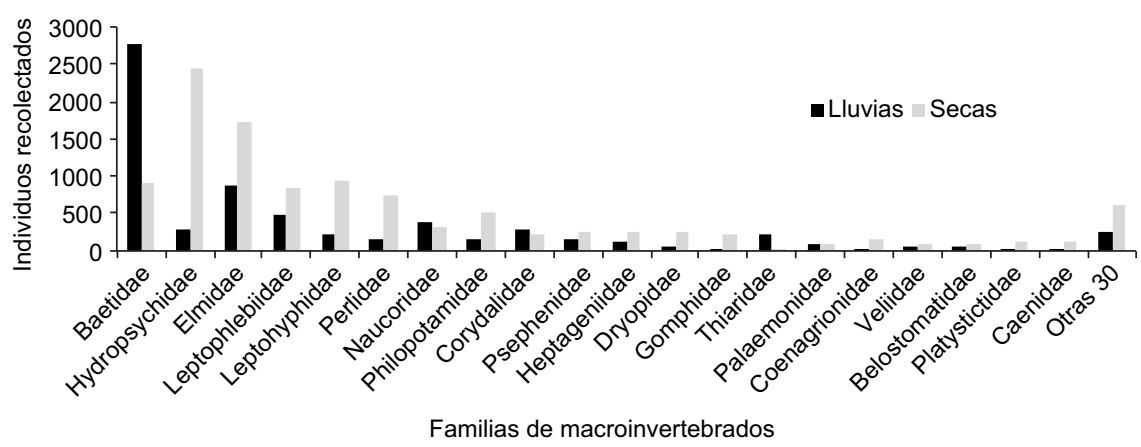

Fig 2. Individuos de macroinvertebrados recolectados por familias en las temporadas de lluvias y secas en la cuenca del río Margaritas. 
C3, Mi1 y C1, mientras que en los sitios M2, M1, M4 se determinó una calidad del agua regular. En temporada de lluvias el sitio M3 presentó el valor de calidad del agua más bajo (59), que corresponde a mala calidad (Cuadro VI).

El análisis de correspondencia canónica (ACC; Fig. 3) indica que la varianza acumulada de la relación entre los parámetros físicos, químicos e hidrológicos del agua y los macroinvertebrados acuáticos se explica por los dos primeros ejes en un $81.1 \%$. Las familias de macroinvertebrados se separan entre sitios y temporadas de muestreo como resultado de la propia estructura de sus comunidades y los parámetros físicos, químicos e hidrológicos del agua. El primer eje explicó el $66.6 \%$ de la variación total. En este eje, el OD y la CE fueron los parámetros con mayor peso en la distribución de las familias de macroinvertebrados. El segundo eje explicó el 14.5\% de la variación total, siendo la VC y el caudal los parámetros que explicaron mejor la distribución de las familias de MA (Fig. 3). En el eje 1, las familias Baetidae y Corydalidae fueron las más relacionadas con el OD, mientras que Hydropsychidae y Gomphidae lo hicieron con la CE. En el eje 2, las familias Thiaridae, Palaemonidae, Philopotamidae y Elmidae se relacionaron con el caudal y la VC, las tres primeras de manera positiva y la última de forma negativa.

\section{DISCUSIÓN}

La cuenca del río Margaritas está afectada principalmente por el cambio de uso del suelo y el aumento de actividades agrícolas y ganaderas hacia las zonas medias y bajas de la cuenca, lo que ha provocado una disminución en la calidad de las riberas como resultado de la reducción y fragmentación de la vegetación ribereña (Tovilla 2005). Esto es similar a lo que sucede en otras cuencas de la zona como la del río Cacaluta (HernándezHernández 2014) en Chiapas, así como en otras cuencas costeras de la vertiente del Pacífico mexicano (Nene-Preciado et al. 2017).

La disminución de la condición ecológica de las riberas propicia una mayor entrada de nutrientes y contaminantes al río con la consecuente disminución de la calidad del agua, especialmente hacia las zonas bajas de la cuenca, lo que podría explicar que en los sitios ubicados a menor altitud dentro de ésta (M3, M2) la calidad del agua haya disminuido en ambas temporadas de muestreo, en relación con las zonas altas. En general, se ha demostrado que los ríos con amplias zonas de amortiguamiento de vegetación ribereña son más efectivos para mantener y mejorar la biodiversidad acuática y la calidad del agua (ÍñiguezArmijos et al. 2014).

CUADRO VI. ÍNDICES BMWP CR Y DE DIVERSIDAD (H') DE FAMILIAS DE MACROINVERTEBRADOS IDENTTIFICADAS EN LOS SITIOS MUESTREADOS DEL RÍO MARGARITAS.

\begin{tabular}{lcccccccc}
\hline Sitios* & \multicolumn{2}{c}{ Individuos } & \multicolumn{2}{c}{ Familias } & \multicolumn{2}{c}{ BMWP_CR** } & \multicolumn{2}{c}{ H' $^{*}$} \\
\cline { 2 - 8 } & Lluvias & Secas & Lluvias & Secas & Lluvias & Secas & Lluvias & Secas \\
\hline L1 & 578 & 253 & 23 & 26 & 103 & 125 & 2.2 & 2.6 \\
Mi1 & 505 & 1058 & 22 & 35 & 117 & 163 & 2.1 & 2.1 \\
L2 & 905 & 538 & 23 & 31 & 106 & 144 & 1.8 & 2.6 \\
Mi2 & 326 & 841 & 22 & 33 & 93 & 133 & 2.3 & 2.2 \\
R1 & 782 & 529 & 26 & 24 & 105 & 112 & 2.2 & 2.5 \\
R2 & 331 & 549 & 21 & 25 & 89 & 108 & 2.2 & 2.4 \\
C1 & 756 & 963 & 25 & 32 & 111 & 154 & 2.0 & 2.7 \\
C2 & 793 & 662 & 20 & 31 & 97 & 122 & 1.0 & 2.8 \\
C3 & 921 & 2398 & 26 & 30 & 121 & 124 & 1.8 & 2.5 \\
M4 & 316 & 584 & 17 & 27 & 72 & 112 & 1.6 & 2.7 \\
M1 & 209 & 1397 & 22 & 30 & 97 & 135 & 2.4 & 2.3 \\
M3 & 149 & 474 & 17 & 24 & 59 & 99 & 1.7 & 2.5 \\
M2 & 150 & 706 & 19 & 23 & 90 & 96 & 2.3 & 2.5 \\
\hline
\end{tabular}

BMWP_CR: Índice del Grupo de Trabajo de Monitoreo Biológico adaptado para Costa Rica.

*El nombre completo de cada sitio se detalla en el Cuadro I; **parámetros del índice BMWP_CR: > 120: excelente calidad de agua; 101-120: buena calidad del agua, no contaminada ni sensiblemente alterada; 61100: calidad regular del agua, contaminación moderada; 36-60: mala calidad del agua, contaminada; 16-35: mala calidad del agua, muy contaminada; < 15: muy mala calidad del agua, extremadamente contaminada. 
Eje 2, $14.5 \%$

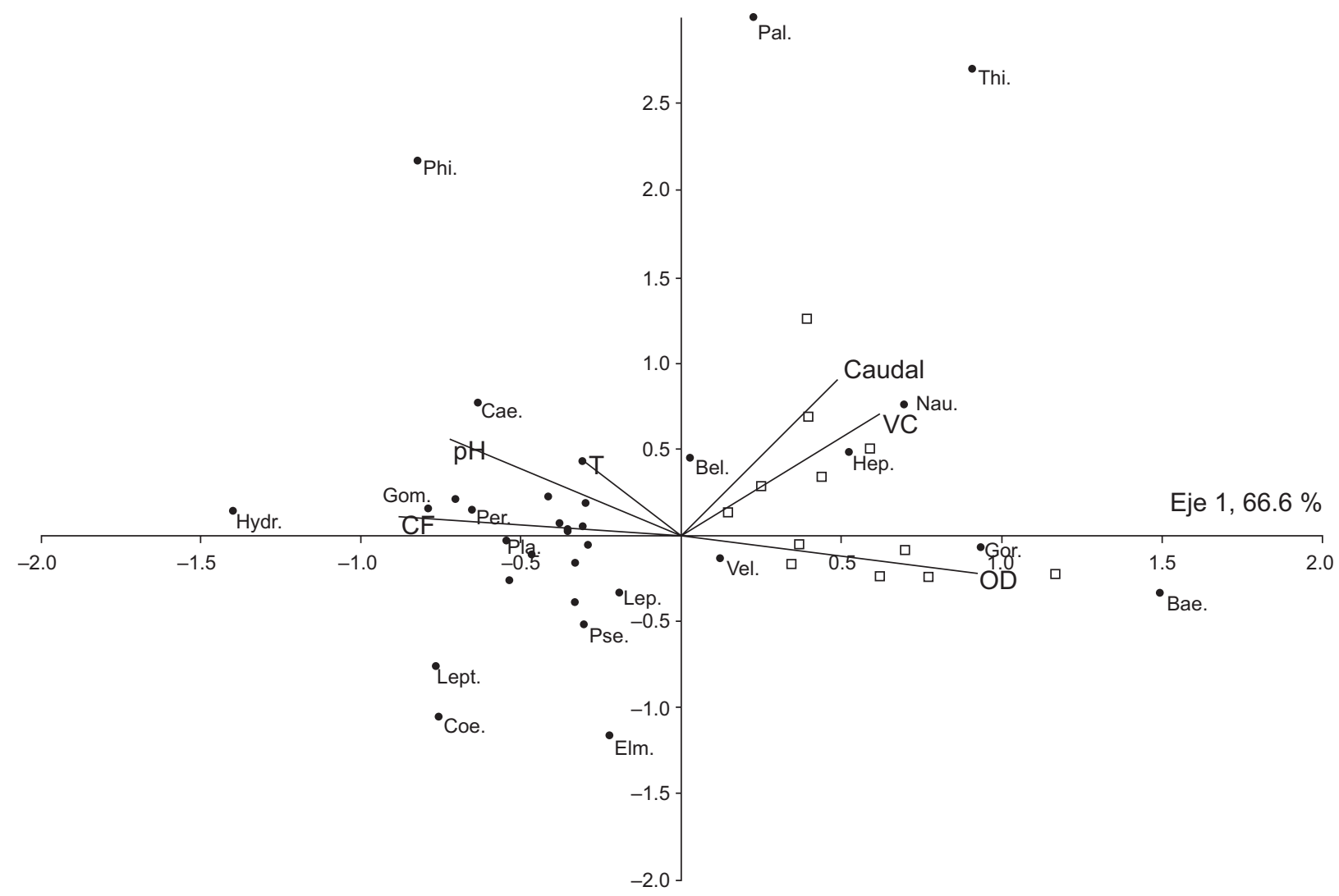

Fig 3. Análisis de correspondencia canónica (ACC) para la presencia de las 20 familias más abundantes de macroinvertebrados acuáticos muestreadas en temporada seca (círculos negros) y de lluvias (cuadrados) en relación con los parámetros físicos y químicos ( $\mathrm{pH}$, temperatura, oxígeno disuelto y conductividad eléctrica) e hidrológicos (caudal y velocidad de la corriente). Las abreviaturas para las familias de macroinvertebrados se explican en el Cuadro III.

La realización de actividades ganaderas en las zonas adyacentes a los sitios con mala y pobre condición ribereña, afecta el balance de los sedimentos y la disminución de la regeneración natural de las especies de plantas ribereñas leñosas, provocando la destrucción del hábitat de la ribera. Lo anterior explica los resultados obtenidos en cuanto a calidad en estos sitios y las diferencias de calidad del agua entre los sitios muestreados. Se ha demostrado que el deterioro del hábitat ribereño, así como la vegetación ribereña, pueden afectar directa e indirectamente a la calidad del agua y las comunidades acuáticas asociadas (Fierro et al. 2017) mediante el aumento de sólidos disueltos en agua, lo que puede afectar la penetración de la luz, aumentar la $\mathrm{T}$ del agua y contribuir a una eutrofización excesiva (Baillie et al. 2005).

En este estudio, los sitios ubicados a menor altitud fueron los más degradados. Sin embargo, algunos sitios (como R2, Mi2, L2 y C2) ubicados en la parte alta de la cuenca muestran signos de degradación, lo que se atribuye a los usos del suelo, principalmente la ganadería, la disminución en la cobertura adyacente a las riberas (pastizal), así como a la presencia de una comunidad humana aledaña al sitio C2, la cual está construida prácticamente en las márgenes del río. La alteración antrópica regularmente es más extensa en altitudes menores (Forio et al. 2017) debido a que ahí los ríos tienen grandes planicies de inundación, lo que permite el desarrollo de riberas más extensas que a su vez favorecen los usos antrópicos, por ejemplo el agrícola.

El uso del suelo y el tipo de cobertura de vegetación adyacente al río están relacionados con la condición de la ribera (Díaz-Pascacio et al. 2018, Ortiz 2019) y son factores determinantes de la calidad del agua superficial y el estado de las comunidades acuáticas (Mesa 2010, Ruiz-Picos et al. 2016). Ambos explican las diferencias registradas entre los sitios de 
este estudio en cuanto a calidad del agua, que fue mejor en sitios con vegetación de selva y secundaria (Mi1, L2, C1) que en sitios con pastizal y la presencia de alguna localidad humana (M3, M2, véase el Cuadro I). Nuestros resultados concuerdan con los de Damanik-Ambarita et al. (2016), quienes encontraron que la calidad del agua en los sitios localizados en bosque fue mejor que en sitios localizados alrededor de zonas residenciales.

Los resultados obtenidos con el BMWP CR en esta investigación indicaron que la calidad del agua en temporada de secas fue mejor que en lluvias, lo cual se explica porque en la primera se registró mayor riqueza y diversidad de MA que en la segunda, además de que se desconoce el valor de la sensibilidad a la contaminación de algunas familias de MA que se registraron en este estudio. Estos resultados contrastan con lo encontrado por Auquilla et al. (2006), quienes registraron que la calidad del agua mejoró durante la temporada de lluvias en la subcuenca del río Jabonal en Costa Rica, lo cual atribuyeron al efecto de la dilución de contaminantes durante esta temporada.

El aumento de la riqueza y diversidad biológicas en temporada de secas es característico de los ambientes lóticos tropicales, donde es común observar esta tendencia. Así lo reportaron para ríos panameños Helson y Williams (2013), quienes registraron que la temporada de secas generalmente tiene valores métricos de invertebrados más altos que la de lluvias. Esta variación entre temporadas se debe a que durante las secas la estabilidad de los sustratos colonizados es mayor, mientras que hay una menor pérdida de material vegetal y una disminución de las corrientes fuertes que pueden arrastrar a los organismos (Auquilla et al. 2006). Además, porque en lluvias existen mayor mortalidad y alteraciones del hábitat debido a las crecidas que ocurren durante esta temporada (Jacobsen et al. 2008).

Sin embargo, algunas familias de MA aumentan su abundancia durante la temporada de lluvias, como se registró en este estudio para la familia Baetidae (Fig. 2). Ésta es una de las familias más diversas y abundantes de macroinvertebrados, con preferencia por sustratos específicos y sensibilidad a la contaminación e impactos antrópicos (Forero-Céspedes et al. 2016). Estos individuos se aferran a las piedras en corrientes rápidas y en las cascadas (Flowers y de la Rosa 2010), lo cual explica su abundancia durante la temporada de lluvias y el que haya sido la familia más abundante del estudio (Domínguez et al. 2009). La familia Hydropsychidae fue la segunda en abundancia, destacando durante la temporada de secas probablemente por la mayor disponibilidad de hábitats. Las especies de esta familia tienen la capacidad de sobrevivir en diferentes tipos de hábitats que le ofrezcan el sustrato necesario, como rocas, piedras, hojas, arena y grava (Forero-Céspedes et al. 2013).

La evaluación de la calidad del agua en el río Margaritas en términos de la diversidad de macroinvertebrados puede ser una opción para otros ríos de la zona. Sin embargo, la escasa investigación que existe sobre los macroinvertebrados puede limitar su uso. Tomando en cuenta lo anterior se recomienda que en los estudios iniciales en otros ríos de la zona se emplee este índice junto con la concentración de contaminantes (Ruiz-Picos et al. 2017). Lo anterior contribuiría a determinar el valor de la sensibilidad a la contaminación de algunas familias de macroinvertebrados, por ejemplo Psephenidae, Pelecorhynchidae e Hydrometridae (Cuadro I), las cuales no están contempladas en el BMWP_CR porque no están presentes en Costa Rica. Este tipo de estudios, sumados a lo reportado aquí, permitirían proponer, mediante la validación y calibración, un índice BMWP para su uso en la zona, donde se incluyan las familias de macroinvertebrados presentes y el valor de su sensibilidad a la contaminación, de manera similar a lo que hicieron Ruiz-Picos et al. (2017) para la zona central de México mediante el estudio de las cuencas de los ríos Apatlaco y Chalma-Tembembe.

Éste es el primer reporte de la diversidad y abundancia de MA acuáticos en el río Margaritas y en la zona costera de Chiapas. En este estudio se contabilizaron un total de 17676 organismos pertenecientes a 13 órdenes y 50 familias, siendo la clase Insecta la más representada. Esto es similar a lo reportado por Forero-Céspedes et al. (2013) para el río Opia en Colombia y ligeramente inferior a las 63 familias reportadas por Ruiz-Picos et al. (2016) en la cuenca del río Balsas, en el centro de México.

La riqueza de invertebrados en los ríos está estructurada puntualmente por eventos históricos y por las condiciones físicas y químicas únicas de cada lugar (Vinson y Hawkins 1998). Esto explica las diferencias puntuales entre los sitios muestreados, ya que aspectos como el caudal, la $\mathrm{VC}$, el $\mathrm{pH}$, el OD, la $\mathrm{T}$, la $\mathrm{CE}$ y la calidad de la ribera varían entre los sitios de esta investigación, lo cual puede generar diferencias en los ensambles de macroinvertebrados (Hershey et al. 2010).

La conductividad eléctrica (CE) es una medida de la capacidad del agua para conducir una corriente eléctrica. Este parámetro es sensible a las variaciones de sólidos disueltos, principalmente de sales minerales. En un cuerpo de agua específico, la CE 
se relaciona con los sólidos totales disueltos y los iones mayoritarios que presentes en el cuerpo de agua (Chapman 1996). La CE de la mayoría de las aguas dulces naturales se encuentra entre 10 y 350 $\mu \mathrm{S} / \mathrm{cm}$ (Chapman 1996); los valores obtenidos en los sitios muestreados en el río Margaritas se encuentran en este rango, lo que indica que el río Margaritas presenta buena calidad del agua en todos los sitios estudiados.

La calidad del agua se refiere a sus características para un determinado uso (Ortiz et al. 2019). Tomando en cuenta los resultados obtenidos aquí para la CE, el agua de los sitios muestreados se clasifica como de salinidad baja y sería apta para el riego agrícola. Richards (1990) menciona que para fines de diagnóstico y clasificación del agua usada para el riego agrícola se debe tomar en cuenta la concentración total de sales solubles (con el objetivo de prevenir la salinización de los suelos) y se puede expresar en términos de la CE. Este autor define cuatro clases de agua: agua de salinidad baja $(\mathrm{C} 1:<250 \mu \mathrm{S} / \mathrm{cm})$, agua de salinidad media (C2: 250-750 $\mu \mathrm{S} / \mathrm{cm})$, agua de salinidad alta (C3: $750-2250 \mu \mathrm{S} / \mathrm{cm}$ ) y agua de salinidad muy alta (C4: $>2250 \mu \mathrm{S} / \mathrm{cm})$.

En condiciones naturales, la concentración iónica expresada como CE disminuye en el periodo de lluvias por la dilución que ocurre al incrementarse el caudal del río y en sequía aumenta por la disminución del caudal (Ortiz et al. 2019). Lo anterior puede explicar los valores obtenidos, los cuales fueron menores durante la temporada de lluvias y mayores en secas. Esto concuerda con lo demostrado por Sabater et al. (2009), quienes afirman que los valores de la CE se relacionan con los caudales en los ríos.

Las respuestas de los MA a la CE son variadas; se han reportado familias indicadoras de bajas concentraciones de este parámetro, por ejemplo Baetidae, Leptohyphidae, Elmidae y Coenagrionidae. Sin embargo, otras familias como Hydroptilidae y Sphaeriidae se han registrado en aguas con valores elevados de CE (Azrina et al. 2006). Esta variación de la sensibilidad de los MA a diferentes contaminantes permite su amplio uso como bioindicadores (Prat et al. 2009). Al respecto, Carvacho (2012) encontró que la CE es uno de los parámetros que determinan el patrón de distribución de los MA en los ríos de la cuenca Limari en Chile.

$\mathrm{El} \mathrm{pH}$ es otro de los parámetros que afectan de manera esencial los procesos biológicos, ya que muchos organismos sobreviven en un intervalo específico (Kannel et al. 2007). Por ejemplo, según Pérez-Castillo y Rodríguez (2008), en el intervalo de 6.5 a 8.5 el agua es apropiada para la subsistencia de comunidades biológicas (p. ej., los MA), mientras que valores mayores a 9.0 y menores de 5.8 producen limitaciones al desarrollo y la fisiología de los organismos acuáticos. Los valores obtenidos en este estudio para ambas temporadas de muestreo estuvieron dentro de los niveles considerados normales para aguas naturales (Pérez-Castillo y Rodríguez 2008).

De manera general, los valores del $\mathrm{pH}$ obtenidos fueron alcalinos. El origen de esta alcalinidad puede ser la presencia de iones bicarbonato $\left(\mathrm{HCO}_{3}{ }^{-}\right)$ aportados al sistema por la disolución de rocas y suelos (Drever 1988). Específicamente, en los sitios muestreados dentro de la cuenca del río Margaritas predomina el tipo de suelo litosol (INEGI 2002), el cual contiene una gran cantidad de material calcáreo que al disolverse forma los iones bicarbonato. Los ríos tienen cierta capacidad para resistir los cambios de $\mathrm{pH}$, lo cual ocurre en términos de alcalinidad del sistema, principalmente debido a la presencia de iones bicarbonato. Esto es similar a lo registrado por Beita-Sandí y Barahona-Palomo (2010) en el río Rincón, Península de Osa, Costa Rica.

El OD es uno de los parámetros más importantes para la vida acuática y el funcionamiento ecológico de los ríos (Martínez e Irurtia 2009), ya que su concentración determina las especies que, de acuerdo con su tolerancia y rango de adaptación, pueden sobrevivir en un determinado cuerpo de agua (Sierra 2011). Chapra y Pelletier (2003) establecieron un rangos de 7 a $8 \mathrm{mg} / \mathrm{L}$ como los valores que indican aguas libres de contaminación. Por lo anterior, todos los sitios de muestreo en el río Margaritas presentaron aguas de buena calidad en la temporada de lluvias. Esto contradice lo encontrado con el BMWP_CR, lo cual se explica porque los resultados del muestreo de parámetros físicos y químicos es puntual mientras que la composición y la diversidad de macroinvertebrados puede deberse a eventos que han ocurrido con anterioridad al día del muestreo, incluso semanas antes. Esto se debe a que los macroinvertebrados, después de una perturbación, necesitan un tiempo mínimo de recolonización de aproximadamente un mes o más (Alba-Tercedor 1996).

La capacidad de los macroinvertebrados para responder a los cambios que ocurren en las condiciones físicas y químicas del agua, así como su rápida respuesta a presiones antrópicas y su bajo costo es lo que ha determinado su amplio uso como indicadores de la calidad del agua (Roldán 1999). En contraste, en la temporada de secas se encontraron valores por debajo del rango óptimo en siete sitios, lo cual puede estar relacionado con el aumento de la $\mathrm{T}$ del agua durante la temporada de secas, lo cual influye en la disminución de la concentración de OD en el agua 
(Harvey et al. 2011). Además, estos valores pueden explicarse por la disminución del caudal y la velocidad de la corriente (VC) en secas lo cual provoca una menor aireación del agua que es característico para esta temporada (Zhen-Wu 2010).

La $\mathrm{T}$ del agua influye en los procesos fisiológicos de los organismos, tales como la respiración microbiana, que es en gran parte responsable de la autopurificación que ocurre en los cuerpos de agua (Chapman 1996). A pesar de que no hay un estándar oficial para la $\mathrm{T}$ del agua (Rasoloariniaina et al. 2015), temperaturas inferiores a $20.2^{\circ} \mathrm{C}$ y superiores a $34.2^{\circ} \mathrm{C}$ son los valores a partir de los cuales el agua se clasifica como mala (Martínez et al. 2013). Los resultados obtenidos en este estudio se encuentran dentro del ámbito normal. Las diferencias observadas para este parámetro entre los muestreos de secas y lluvias son características de las variaciones temporales y se explican porque la cantidad de radiación solar que recibe el agua es mayor en la temporada de secas a causa de la disminución del caudal, por tanto la $\mathrm{T}$ del agua en esta temporada es más alta (Martínez et al. 2013).

Finalmente, es importante destacar que los métodos empleados y los resultados obtenidos en este estudio se pueden utilizar para apoyar la gestión futura del agua en la cuenca del río Margaritas y cuencas similares situadas en los trópicos. Los dos índices utilizados para valorar la calidad de la ribera y la calidad del agua (RQI y BMWP_CR, respectivamente) son fáciles de utilizar en campo y brindan una evaluación eficaz del estado de conservación del sitio.

En cuanto al uso del BMWP_CR, es la primera vez que se emplea para determinar la calidad de agua en ríos de esta zona y en particular en el río Margaritas, por lo que es necesario ampliar la investigación al respecto, pues se encontró mala calidad del agua en la temporada de lluvias. Igualmente, se recomienda crear un índice propio adaptado y calibrado para evaluar la calidad del agua en otros ríos del sureste de México.

El uso del RQI también es novedoso en este río. Existen referencias de su uso en otros ríos en México (Díaz-Pascacio et al. 2018, Ortiz 2019) y reportes de que es una buena alternativa para determinar la condición ecológica de los ríos. Además, el RQI tiene una ventaja adicional, ya que contempla acciones de manejo para los sitios degradados (González del Tánago y García de Jalón 2011). En la región no se han realizado estudios similares, por lo que esta investigación constituye un aporte sustancial al conocimiento de la calidad del agua y de la ribera, así como, al conocimiento de la fauna regional de macroinvertebrados en los ríos de la costa de Chiapas, México.

\section{CONCLUSIONES}

La evaluación de la calidad de las riberas mostró que las condiciones de los sitios variaron de "malas" a "muy buenas". Los sitios con mayores valores de RQI fueron L1 y Mi1, únicos que obtuvieron la condición de "muy bueno", en los cuales se observó vegetación ribereña en buen estado de conservación. Los sitios con menos calidad de las riberas fueron M3 y M2, que fueron clasificados como "malo" y "pobre", respectivamente. En estos sitios se observaron indicios de erosión en las orillas y pérdida de la conectividad transversal del cauce con la llanura de inundación y la conectividad vertical con el medio hiporreico, debido a procesos de urbanización y construcción de caminos y bordos. Los atributos del índice que presentaron mayores afectaciones fueron la regeneración natural y la composición y estructura de la vegetación ribereña.

La calidad del agua del río Margaritas, según los parámetros físicos y químicos del agua, varía a lo largo de su cauce pero son óptimos para el desarrollo de la vida acuática. El pH es alcalino-. Los valores máximos de OD se encontraron durante la temporada de lluvias. La conductividad es mayor durante la temporada de secas y sus valores no exceden el límite permisible para el buen funcionamiento del ecosistema. Los valores de caudal y la velocidad de la corriente están influenciados por la temporada de muestreo (lluvias-secas), lo cual se infiere debido a que los mayores valores se obtuvieron en lluvias para todos los sitios.

En cuanto al índice de calidad de agua BMWP $\mathrm{CR}$, generalmente los valores en secas fueron mayores que en lluvias, lo cual indica que la calidad del agua disminuyó durante la segunda. Esto se atribuye a la menor diversidad de macroinvertebrados registrados en esta temporada y a que se desconoce el valor de la sensibilidad de algunas familias de estos individuos reportadas en este estudio.

En el aspecto biológico se contabilizaron un total de 17676 individuos de macroinvertebrados (10 954 en la temporada de secas y 6722 en lluvias), pertenecientes a 13 órdenes y 50 familias. La familia con mayor cantidad de individuos recolectados fue Baetidae con 3686 individuos, seguida de Hydropsychidae con 2727 y Elmidae con 2596. La riqueza y diversidad biológica $\left(\mathrm{H}^{\prime}\right)$, usando el número de familias de macroinvertebrados sugieren que en la temporada de secas hay mejores índices de diversidad, equidad y abundancia. Los parámetros con mayor influencia en la distribución de familias de macroinvertebrados fueron el OD, la CE, la velocidad de la corriente y el caudal. 


\section{AGRADECIMIENTOS}

Agradecemos al Consejo Nacional de Ciencia y Tecnología (CONACYT) por la beca No. 430094 otorgada para estudios de doctorado a la primera autora de este trabajo. A Juan Juárez-Flores, Cinthia Trinidad-Ocaña y Mayumi Vega-Polanco por su ayuda con la separación e identificación de macroinvertebrados. A Freddy Ángel Sánchez y Sandro Torres Hernández por su ayuda en el trabajo de campo. Al Lic. José Higinio Urbina López por la elaboración del mapa de los sitios de estudio, en el Laboratorio de Análisis de Información Geográfica y Estadística del Colegio de la Frontera Sur, unidad Tapachula.

\section{REFERENCIAS}

Alba-Tercedor J. y Sánchez-Ortega A. (1988). Un método rápido y simple para evaluar la calidad biológica de las aguas corrientes basado en el de Hellawell (1978). Limnetica 4 (1), 51-56.

Alba-Tercedor J. (1996). Macroinvertebrados acuáticos y calidad de las aguas de los ríos. Memorias. IV Simposio del agua en Andalucía (SIAGA). Almería, Andalucía, 203-213.

Alonso A. y Camargo J.A. (2005). Estado actual y perspectivas en el empleo de la comunidad de macroinvertebrados bentónicos como indicadora del estado ecológico de los ecosistemas fluviales españoles. Ecosistemas 14 (3), 87-99. https://doi.org/10.7818/ ECOS.432

Armitage P.D., Moss D., Wright J.T. y Furse M.T. (1983). The performance of the new biological water quality score system based on macroinvertebrates over a wide range of unpolluted running water sites. Water Res. 17 (3), 333-347. https://doi.org/10.1016/00431354(83)90188-4

Arriaga-Gaona M.L. (2009). Monitoreo de la calidad del agua del río Tecolutla desde Coyutla hasta Gutiérrez Zamora, Veracruz. Revista Latinoamericana de Recursos Naturales 5 (2), 141-147.

Auquilla R.C., Astorga Y. y Jiménez-Otárola F. (2006). Influencia del uso del suelo en la calidad del agua en la subcuenca del río Jabonal, Costa Rica. Recursos Naturales y Ambiente 48, 81-92.

Azrina M.Z., Yap C.K., Ismail A.R., Ismail A. y Tan S.G. (2006). Anthropogenic impacts on the distribution and biodiversity of benthic macroinvertebrates and water quality of the Langat River, Peninsular Malaysia. Ecotox. Environ. Safe. 64 (3), 337-347. https://doi. org/10.1016/j.ecoenv.2005.04.003
Baillie B.B., Collier K.J. y Nagels J. (2005). Effects of forest harvesting and woody debris removal on two Northland streams, New Zealand. New Zeal. J. Mar. Fresh. 39 (1), 1-15. https://doi.org/10.1080/0028833 0.2005 .9517290

Beita-Sandí W. y Barahona-Palomo M. (2011). Físicoquímica de las aguas superficiales de la cuenca del río Rincón, Península de Osa, Costa Rica. Cuadernos de Investigación UNED 2 (2), 157-179.

Carvacho C.A. (2012). Estudio de las comunidades de macroinvertebrados bentónicos y desarrollo de un índice multimétrico para evaluar el estado ecológico de los ríos de la cuenca del Limari en Chile. Tesis de Maestría. Facultad de Biología, Universidad de Barcelona, España, 70 pp.

Chang F.H., Lawrence J.E., Ríos-Touma B. y Resh V.H. (2014). Tolerance values of benthic macroinvertebrates for stream biomonitoring: assessment of assumptions underlying scoring systems worldwide. Environ. Monit. Assess. 186, 2135-2149. https://doi. org/10.1007/s10661-013-3523-6

Chapman D.V. (1996). Water quality assessments: A guide to the use of biota, sediments and water in environmental monitoring. 2nd ed. Taylor and Francis Group, Nueva York, EUA, 636 pp.

Chapra S.C. y Pelletier G.J. (2003). QUAL2K: A modeling framework for simulating river and stream water quality: documentation and user's manual. Civil and Environmental Engineering Department, Tufts University, Medford, Massachusetts, $121 \mathrm{pp}$.

Cotler H. (2007). El manejo integral de cuencas en México. Secretaría de Medio Ambiente y Recursos Naturales, Ciudad de México, 348 pp.

CONAGUA (2009). Atlas del agua en México. Manual. Comisión Nacional del Agua, México.

CONAGUA (2011). Identificación de reservas potenciales de agua para el medio ambiente en México. Comisión Nacional del Agua, México, 98 pp.

Czerniawska-Kusza I. (2005). Comparing modified biological monitoring working party score system and several biological indices based on macroinvertebrates for water-quality assessment. Limnologica 35 (3), 169-176. https://doi.org/10.1016/j. limno.2005.05.003

Damanik-Ambarita M.N., Lock K., Boets P., Everaert G., Nguyen T.H.T., Forio M.A.E., Musonge P.L.S., Suhareva N., Bennetsen E., Landuyt D., Dominguez-Granda L., y Goethals P.L.M. (2016). Ecological water quality analysis of the Guayas river basin (Ecuador) based on macroinvertebrates indices. Limnologica 57, 27-59. https://doi.org/10.1016/j.limno.2016.01.001

Díaz-Pascacio E., Ortega-Argueta A., Castillo-Uzcanga M.M. y Ramírez-Marcial N. (2018). Influence of land 
use on the riparian zone condition along an urban-rural gradient on the Sabinal River, Mexico. Bot. Sci. 96 (2), 180-199. https://doi.org/10.17129/botsci.1858

Domínguez E., Molineri C. y Nieto C. (2009). Ephemeroptera. En: Macroinvertebrados bentónicos sudamericanos: sistemática y biología. Vol. 656 (Domínguez E. y Fernández H., Eds.). Fundación Miguel Lillo, Tucumán, Argentina, pp. 55-94.

Drever J.L. (1988). The geochemistry of natural waters: Surface and groundwater environments. Prentice-Hall, Englewood Cliffs, Nueva Jersey, EUA.

Espinal-Carreón T., Sedeño J.E. y López E. (2013). Evaluación de la calidad del agua en la Laguna de Yuriria, Guanajuato, México, mediante técnicas multivariadas: un análisis de valoración para dos épocas: 2005, 20092010. Rev. Int. Contam. Ambie. 29 (3), 147-163.

Fierro P., Bertrán C., Tapia J., Hauenstein E., Peña-Cortés F., Vergara C., Cerna C. y Vargas-Chacoff L. (2017). Effects of local land-use on riparian vegetation, water quality, and the functional organization of macroinvertebrate assemblages. Sci. Total Environ. 609, 724-734. https://doi.org/10.1016/j.scitotenv.2017.07.197

Flowers R.W. y de la Rosa C. (2010). Ephemeroptera. En: Macroinvertebrados de agua dulce de Costa Rica I. Capítulo 4. Rev. Biol. Trop. 58 (Suppl. 4), pp. 63-93.

Forero-Céspedes A.M., Reinoso-Flórez G. y Gutiérrez C. (2013). Evaluación de la calidad del agua del río Opia (Tolima-Colombia) mediante macroinvertebrados acuáticos y parámetros fisicoquímicos. Caldasia 35 (2), 371-387.

Forero-Céspedes A.M., Gutiérrez C. y Reinoso-Flórez G. (2016). Composición y estructura de la familia Baetidae (Insecta: Ephemeroptera) en una cuenca andina colombiana. Hidrobiológica 26 (3), 459-474. https://doi. org/10.24275/uam/izt/dcbs/hidro/2016v26n3/Forero

Forio M.A.E., Mouton A., Lock K., Boets P., Nguyen T.H.T., Damanik-Ambarita M.N., Musonge P.L.S., Dominguez-Granda L. y Goethals P. L. (2017). Fuzzy modelling to identify key drivers of ecological water quality to support decision and policy making. Environ. Sci. Policy. 68, 58-68. https://doi.org/10.1016/j. envsci.2016.12.004

García E. (1998). Climas (clasificación de Köppen, modificado por García). Cobertura digital escala 1:1000000. Comisión Nacional para el Conocimiento y Uso de la Biodiversidad, México.

González del Tánago M. y García de Jalón D. (2011). Riparian Quality Index (RQI): A methodology for characterising and assessing the environmental conditions of riparian zones. Limnetica 30 (2), 235-254. https:// doi.org/10.23818/limn.30.18

Gutiérrez-Fonseca P.E. y Lorion C.M. (2014). Application of the BMWP-Costa Rica biotic index in aquatic biomonitoring: Sensitivity to collection method and sampling intensity. Rev. Biol. Trop. 62 (Suppl. 2), 275-289. https://doi.org/10.15517/RBT.V62I0.15792

Hammer O., Harper D.A.T. y Ryan P.D. (2012). PASTPalaeontological Statistics (version 3.21.) [en línea]. http://folk.uio.no/ohammer/past 09/10/2018

Harvey R., Lye L., Khan A. y Paterson, R. (2011). The influence of air temperature on water temperature and the concentration of dissolved oxygen in Newfoundland rivers. Can. Water Resour. J. 36 (2), 171-192. https:// doi.org/10.4296/cwrj3602849

Helson J.E. y Williams D.D. (2013). Development of a macroinvertebrate ultimetric index for the assessment of low-land streams in the neotropics. Ecol. Indic. 29, 167-178. https://doi.org/10.1016/j. ecolind.2012.12.030

Hernández-Hernández J.L. (2014). Caracterización de uso de suelo y evaluación de la calidad riparia del río Cacaluta, Acacoyagua, Chiapas, México. Tesis de Maestría. El Colegio de la Frontera Sur, Tapachula, Chiapas, México.

Hershey A.E., Lamberti G.A., Chaloner D.T. y Northington R.M. (2010). Aquatic insect ecology. En: Ecology and classification of North American freshwater invertebrates (Thorp J.H. y Covich A.P., Eds.). Academic Press, Londres, 659-694.

Hood W.G. y Naiman R.J. (2000). Vulnerability of riparian zones to invasion by exotic vascular plants. Plant Ecol. 148 (1), 105-114. https://doi. org/10.1023/A:1009800327334

INEGI (2002). Conjunto de datos vectoriales edafológico. Escala 1:250000. Serie III (continuo nacional). Instituto Nacional de Estadística y Geografía, México.

INEGI (2013). Conjunto de datos vectoriales de la carta de uso del suelo y vegetación. Escala 1:250000. Serie V (continuo nacional). Instituto Nacional de Estadística y Geografía, México.

Íñiguez-Armijos C., Leiva A., Hans-Georg F., Hampel H. y Breuer L. (2014). Deforestation and benthic indicators: how much vegetation cover is needed to sustain healthy Andean streams? PLoS One 9 (8), 1-10. https:// doi.org/10.1371/journal.pone.0105869

Jacobsen D., Cressa C., Mathooko J.M. y Dudgeon D. (2008). Macroinvertebrates. Composition, life histories and production. En: Tropical stream ecology (Dudgeon D., Ed.). Academic Press, Londres, 66-105. https://doi. org/10.1016/B978-012088449-0.50006-6

Kannel P.R., Lee S., Soo Lee Y., Kanel S.R. y Khan S. (2007). Application of water quality indices and dissolved oxygen as indicators for river water classification and urban impact assessment. Environ. Monit. Assess. 132 (1-3), 93-110. https://doi. org/10.1007/s10661-006-9505-1 
Karr J.R. (1999). Defining and measuring river health. Freshwater Biol. 41 (2), 221-234. https://doi. org/10.1046/j.1365-2427.1999.00427.x

Magdaleno F., Martínez R. y Roch V. (2010). Índice RFV para la valoración del estado del bosque de ribera. Ingeniería Civil 157, 85-96.

Martínez J.P. e Irurtia A.E. (2009). El marco físico: la cuenca. En: Conceptos y técnicas en ecología fluvial. (Sabater S. y Elosegui A., Eds.). Fundación BBVA, Girona, España, 39-50.

Martínez G., Fermín I., Brito F., Márquez A., de La Cruz R., Rodríguez G., Hernández D., Parra E., González M.J., Márquez A. y Pinto F. (2013). Calidad de las agua del Caño Mánamo, delta del río Orinoco, Venezuela. Boletín del Instituto Oceanográfico de Venezuela 52 (1), 17-27.

Merritt R.W., Cummins K.W. y Berg M.B. (2008). An introduction to the aquatic insects of north america. $4 \mathrm{a}$ ed. Kendall Hunt Publishing, Dubuque, Iowa, 1158 pp.

Mesa L.M. (2010). Effect of spates and land use on macroinvertebrate community in neotropical Andean streams. Hydrobiologia 641 (1), 85-95. https://doi. org/10.1007/s10750-009-0059-4

Moreno C.E. (2001). Métodos para medir la biodiversidad. Manuales y Tesis SEA. Vol. 1. Zaragoza, España, 84 pp.

Naiman R.J., Décamps H. y Pollock M. (1993). The role of riparian corridors in maintaining regional biodiversity. Ecol. Appl. 3, 209-212. https://doi. org/10.2307/1941822

Naiman R.J. y Décamps H. (1997). The ecology of interfaces: Riparian zones. Annu. Rev. Ecol. Syst. 28 (1), 621-658. https://doi.org/10.1146/annurev.ecolsys.28.1.621

Nene-Preciado A.J., Sansón G.G., Mendoza M.E. y Bátiz F.D.A.S. (2017). Cambio de cobertura y uso de suelo en cuencas tropicales costeras del Pacífico central mexicano. Investigaciones Geográficas (94), 64-81. https://doi.org/10.14350/rig.56770

Ortiz A.C.I. (2019). Los corredores ribereños en la cuenca del río Ayuquila-Armería en México. Vegetación ribereña y calidad de las riberas. Centro Universitario de la Costa Sur, Universidad de Guadalajara. Guadalajara, México.

Ortiz M.I., Can Á., Romero C.A., Cruz E. y Madueño A. (2019). Calidad del agua para uso agrícola del río Mololoa, México. Terra Latinoamericana 37 (2), 185195. https://doi.org/10.28940/terra.v37i2.406

Pardo I., Álvarez M., Casas J., Moreno J.L., Vivas S., Bonada N., Alba-Tercedor J., Jáimez-Cuéllar P., Moyà G. y Prat N. (2002). El hábitat de los ríos mediterráneos. Diseño de un índice de diversidad de hábitat. Limnetica 21 (2), 115-133. https://doi.org/10.23818/ limn. 21.21
Pérez-Castillo A.G. y Rodríguez A. (2008). Índice fisicoquímico de la calidad de agua para el manejo de lagunas tropicales de inundación. Rev. Biol. Trop. 56 (4), 1905-1918. https://doi.org/10.15517/RBT. V56I4.5769

Pinilla G.A. (2000). Indicadores biológicos en ecosistemas acuáticos continentales de Colombia. Fundación Universidad de Bogotá Jorge Tadeo Lozano, Bogotá, Colombia, 67 pp.

Poder Ejecutivo (2007). Reglamento para la evaluación y clasificación de la calidad de cuerpos de agua superficiales 33903-MINAE-S. Decreto N. 33903-MINAE-S. Gaceta N. 178. San José, Costa Rica, 17 de septiembre.

Prat N., Ríos B., Acosta R. y Rieradevall, M. (2009). Los macroinvertebrados como indicadores de calidad de las aguas. En: Macroinvertebrados bentónicos sudamericanos. Sistemática y biología. (Domínguez E. y Fernández H.R., Eds.). Fundación Miguel Lillo, Tucumán, Argentina, 631-654.

QGIS (2019). Sistema de Información Geográfica QGIS. Proyecto de Fundación Geoespacial de Código Abierto (versión 3.10.) [en línea]. https://qgis.org/es 10/11/2019

Rasoloariniaina J.R., Ganzhorn, J.U. y Raminosoa, N. (2015). Physicochemical and bacteriological water quality across different forms of land use on the Mahafaly Plateau, Madagascar. Water Qual. Expos. Hea. 7 (2), 111-124. https://doi.org/10.1007/s12403014-0129-3

Richards L.A. (1990). Diagnóstico y rehabilitación de suelos salinos y sódicos. Manual No. 60. Departamento de Agricultura de los Estados Unidos de America. Limusa, México, D.F.

Roldán G. (1999). Los macroinvertebrados y su valor como indicadores de la calidad del agua. Rev. Acad. Colomb. Cienc. Ex. Fis. Nat. 23 (88), 375-387.

Ruiz-Picos R.A., Sedeño-Díaz J.E. y López-López E. (2016). Ensambles de macroinvertebrados acuáticos relacionados con diversos usos del suelo en los ríos Apatlaco y Chalma-Tembembe (cuenca del Río Balsas), México. Hidrobiologica 26 (3), 443-458. https://doi. org/10.24275/uam/izt/dcbs/hidro/2016v26n3/Lopez

Ruiz-Picos R.A., Kohlmann B., Sedeño-Díaz J.E. y López-López E. (2017). Assessing ecological impairments in Neotropical rivers of Mexico: Calibration and validation of the Biomonitoring Working Party Index. Int. J. Environ. Sci. Te. 14 (9), 1835-1852. https://doi. org/10.1007/s13762-017-1299-x

Ruiz N.E.S., Escobar Y.C. y Escobar J.C. (2007). Revisión de parámetros fisicoquímicos como indicadores de calidad y contaminación del agua. Ingeniería e Investigación 27 (3), 172-181. 
Sabater S., Donato J., Giorgi A. y Elosegui A. (2009). El río como ecosistema. En: Conceptos y técnicas en ecología fluvial (Sabater S. y Elosegui A., Eds.). Fundación BBVA, Girona, España, 23-37.

Sierra C.A. (2011). Calidad del agua. Evaluación y diagnóstico. Universidad de Medellín, Colombia, 457 pp.

Springer M. (2010). Biomonitoreo acuático. Rev. Biol. Trop. 58 (suppl. 4), 53-59. https://doi.org/10.15517/ RBT.V58I4.20082

Thompson F.G. (2004). An identification manual for the freshwater snails of Florida. Florida Museum of Natural History, Gainesville, $92 \mathrm{pp}$.

Tovilla C. (2005). Agonía y desaparición de los ríos y humedales en la costa de Chiapas. Ecofronteras 25, 5-8.

Tyagi S., Sharma B., Singh P. y Dobhal R. (2013). Water quality assessment in terms of water quality index. Am. J. Water Res. 1 (3), 34-38. https://doi.org/10.12691/ ajwr-1-3-3

Vinson M. R. y Hawkins C.P. (1998). Biodiversity of stream insects: Variation at local, basin, and regional scales. Annu. Rev. Entomol. 43 (1), 271-293. https:// doi.org/10.1146/annurev.ento.43.1.271
Vivas S., Casas J., Pardo I., Robles S., Bonada N., Mellado, A., Prat N., Alba-Tercedor J., Álvarez M., Bayo M.M., Jáimez-Cuéllar P., Suárez M.L., Toro M., Vidal-Abarca M.R., Zamora-Muñoz C. y Moyá G. (2002). Aproximación multivariante en la exploración de la tolerancia ambiental de las familias de macroinvertebrados de los ríos mediterráneos del proyecto GUADALMED. Limnetica 21 (3-4), 149-173.

Vorosmarty C.J., McIntyre P.B., Gessner M.O., Dudgeon D., Prusevich A., Green P., Glidden S., Bunn S.E., Sullivan C.A., Liermann C.R. y Davies P.M. (2010). Global threats to human water security and river biodiversity. Nature 467 (7315), 555-561. https://doi. org/10.1038/nature09440

Wetzel R. y Likens G. (2000). Limnological analyses, Springer Verlag, Nueva York, EUA, 429 pp.

Wiggins G.B. (2000). Larvae of North America caddisfly genera (Trichoptera). University of Toronto Press, Toronto, Canadá, 452 pp.

Zhen-Wu B.Y. (2010). Índices de calidad del agua en la microcuenca de la quebrada Victoria, Guanacaste, Costa Rica (2007-2008). UNED Research Journal 2 (1), 45-61. https://doi.org/10.22458/urj.v2i1.221 